九州大学学術情報リポジトリ

Kyushu University Institutional Repository

Interactive Evolutionary Computation: Fusion of the Capabilities of EC Optimization and Human Evaluation

Takagi, Hideyuki

Department of Art and Information Design, Kyushu University

http://hdl. handle. net/2324/1670053

出版情報：Proceedings of the IEEE. 89 (9)，pp.1275-1296，2001-09-09. IEEE バージョン :

権利関係 : 


\title{
Interactive Evolutionary Computation: Fusion of the Capabilities of EC Optimization and Human Evaluation
}

\author{
Hideyuki Takagi, Member, IEEE
}

\begin{abstract}
In this paper, we survey the research on interactive evolutionary computation (IEC). The IEC is an EC that optimizes systems based on subjective human evaluation. The definition and features of the IEC are first described and then followed by an overview of the IEC research. The overview primarily consists of application research and interface research. In this survey, the IEC application fields include graphic arts and animation, 3-D CG lighting, music, editorial design, industrial design, facial image generation, speech processing and synthesis, hearing aid fitting, virtual reality, media database retrieval, data mining, image processing, control and robotics, food industry, geophysics, education, entertainment, social system, and so on. Also in this survey, the interface research to reduce human fatigue includes improving fitness input interfaces and displays based on fitness prediction, accelerating EC convergence especially in early EC generations, examining combinations of interactive and normal EC, and investigating active user intervention. Finally, we discuss the IEC from the point of the future research direction of computational intelligence. In order to show the status quo IEC research, this paper primarily features a survey of about 250 IEC research papers rather than a carefully selected representation of a few papers.
\end{abstract}

Keywords - interactive evolutionary computation, survey, applications, interface

\section{INTRODUCTION}

There are two types of target systems for system optimization: systems whose optimization performances are numerically - or at least quantitatively - defined as evaluation functions and systems whose optimization indexes are difficult to specify. Most engineering research uses several optimization methods based on minimizing error criteria and focus on the former, which includes auto-control, pattern recognition, engineering design, and so on.

However, to obtain the most favorable outputs from interactive systems that create or retrieve graphics or music, such outputs must be subjectively evaluated. It is difficult, or even impossible, to design human evaluation explicit functions. Generally, the best system outputs such as images, acoustic sounds, and virtual realities can be detected by the human senses and be evaluated from the user's impressions, preferences, emotions, and understanding. There are many systems, not only in the artistic or aesthetic fields, but also in the engineering and education fields as described later in this paper. Their system param-

Hideyuki Takagi is with Dept. of Art and Information Design, Kyushu University, takagi@design.kyushu-u.ac.jp, URL: http://www.design.kyushu-u.ac.jp/ takagi/. This paper is a draft for: Hideyuki Takagi, "Interactive Evolutionary Computation: Fusion of the Capacities of EC Optimization and Human Evaluation," Proceedings of the IEEE, vol.89, no.9, pp.1275-1296 (2001). eters or structures must be optimized based on the user's subjective evaluation. Since we cannot use the gradient information of our mental psychological space, we need another approach that is different from conventional optimization methods.

Interactive Evolutionary Computation (IEC) is an optimization method that adopts evolutionary computation (EC) among system optimization based on subjective human evaluation. It is simply an EC technique whose fitness function is replaced by a human user. Fig. 1 shows a general IEC system where a user sees or hears and evaluates system outputs and the EC optimizes the target system to obtain the preferred output based on the user's evaluation. In this sense, we can say that the IEC is a technology that embeds human preference, intuition, emotion, psychological aspects, or a more general term, KANSEI, in the target system.

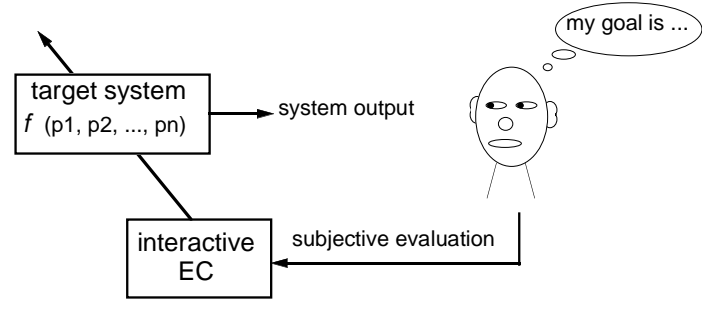

Fig. 1. General IEC system: system optimization based on subjective evaluation.

There are two main definitions of IEC. The narrow definition of IEC is "the technology that EC optimizes the target systems based on subjective human evaluation as fitness values for system outputs." The broader definition of IEC is "the technology that EC optimizes the target systems having an interactive human-machine interface." A previous definition, "an EC technique whose fitness function is replaced with a human user," is of the former. We surveyed only IEC papers of the narrow definition in this paper because most papers that appeal to the IEC aspect are categorized by the narrow definition although there are some exceptions [36], [37], [62], [111]. However, there are many EC-based human-machine interaction papers that do not explicitly appeal to the IEC aspect. Note that the discussion on human interface in section IV is useful for the IEC research based on the broader definition, too.

Next, we explain the terms used in this paper. EC is a biologically inspired general computational concept and includes genetic algorithms (GA), evolutionary strategy 


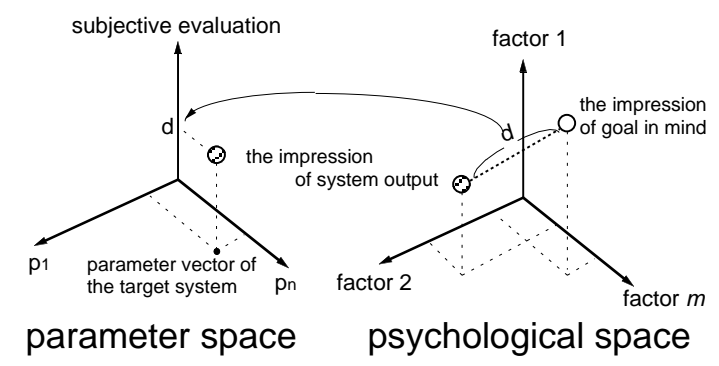

Fig. 2. Psychological distance between target in our psychological spaces and actual system outputs become the fitness axis of a feature parameter space where EC searches for the global optimum in an IEC system.

(ES), genetic programming (GP), and evolutionary programming (EP). We use the term of interactive GA, ES, GP, and EP (IGA, IES, IGP, and IEP, respectively) to individually explain each IEC research in this paper. The subjective evaluation of the IEC is generally in $n$ levels of rating, and the minimum evaluation scale level is two levels, meaning to select and not select EC individuals. This type of the IEC is called simulated breeding or user selection because of the analogy to artificial mating. The term of individual is also frequently used. The EC is a population-based searching algorithm and outputs multiple candidates, each called an individual, as system outputs. See EC textbooks for other terms and concepts for further details.

The technical framework of the IEC is described in section II, and an IEC survey follows in sections III and IV. The biggest feature of this paper is the broad survey of IEC research rather than careful selection of representative papers. This survey not only includes IEC applications but also IEC interface research, another feature of this paper. Finally, we discuss the future of IEC research from a computational intelligence research perspective.

\section{Technical Framework and Features of IEC}

IEC is a technology that human and EC cooperatively optimize target systems based on a mapping relationship between the feature parameter and psychological spaces. IEC users evaluate individuals according to the distance between the target in their psychological spaces and the actual system outputs, and the EC searches for the global optimum in a feature parameter space according to the psychological distance (see Fig. 2).

Conventional approaches for these human evaluationbased systems have frequently modeled the human evaluation characteristics and embedded the substitute evaluation model in optimization systems. We call this approach be an analytical approach. The analytical approach is a common approach in AI research, but it is difficult to perfectly model, for example, a personal preference model. Conversely, the IEC is based on a synthetic approach that directly embeds a human as a black box evaluator in the optimization system and allows the computer to optimize the target system according to the user evaluation [203],
[216], [218].

IEC users sometimes do not evaluate phenotypes of EC individuals but system outputs specified by the EC individuals. For example, they do not directly evaluate filter coefficients but the images or sounds processed by the filters.

We cannot avoid the fluctuation of human preference, the target coordinate in a psychological space, and human evaluation, the distance measured in the psychological space even if we keep a same scale in mind and do consistent evaluation. The requirement of the IEC is to determine the coordinates in a feature parameter space that is mapped to the neighborhood of the target in a psychological space even if the human's subjective evaluation for same image or sound fluctuates according to time. Fortunately, it is reported that an EC search is robust for noise and that there are few influences from the fluctuation through simulation using actual measured human fluctuation characteristics of subjective evaluation [147], [199], [201].

The global optimum of the IEC is very rough because every system output that a human user cannot distinguish are considered to be psychologically the same. The global optimum of the IEC is not a point but rather an area from the normal EC optimization perspective. That is, a wide IEC global optimum area is preferable. For example, suppose we wanted to find the next model of Toyota Camry or Honda Accord. The purpose is not to determine only one point, like as montage facial image of a suspect, but to determine several different car models that possess a common impression based on previous models.

The remaining IEC technical problems need to solve the human fatigue problem that is common to all humanmachine interaction systems and to accelerate EC convergence with a small population size and a few generation numbers, inherently related to the IEC fatigue problem. The EC population size is limited by the number of individual images that are spatially displayed on a computer monitor simultaneously or by the human capacity to remember sounds or images for time-sequentially displayed individual sounds or movies. The number of EC search generations is limited by human fatigue as well, and 10 or 20 EC search generations are usually the maximum number of generations for normal use of the IEC search. Research to solve these problems are introduced in section IV in detail.

It is important not only to expand the IEC application fields but also to evaluate their effectiveness in each field and to develop several IEC interfaces resulting in less fatigue for the practical use of the IEC. Since the IEC involves a human, subjective and statistical tests are essential to evaluate the effectiveness [157]. We cannot expect to develop a practical IEC technology without an objective and quantitative evaluation, even in the field of artistic creativity that seems unrelated to a quantitative evaluation. This is an author's special remark to call IEC researchers' attention. 


\section{TABLE I}

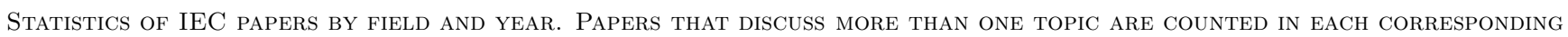
CATEGORY

\begin{tabular}{|c|c|c|c|c|c|c|c|c|c|c|c|c|c|}
\hline & $1980 \mathrm{~s}$ & 1990 & 1991 & 1992 & 1993 & 1994 & 1995 & 1996 & 1997 & 1998 & 1999 & 2000 & total \\
\hline graphic art \& CG animation & 2 & & 3 & 2 & 4 & 5 & 5 & 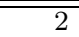 & 2 & 4 & 9 & 4 & $\overline{442}$ \\
\hline 3-D CG lighting design & & & & & & & & 1 & 3 & 1 & & & 5 \\
\hline music & & & & & 1 & 3 & 3 & 1 & & 1 & 3 & 5 & 17 \\
\hline editorial design & & & & & & & & & 1 & & 1 & 2 & 4 \\
\hline industrial design & & & & 2 & 2 & 1 & 5 & 4 & & 2 & 4 & 9 & 29 \\
\hline face image generation & & & 1 & & 1 & 1 & 2 & & 1 & 4 & 5 & 1 & 16 \\
\hline speech processing \& prosodic control & & & & & & & 2 & 1 & 2 & & 1 & 1 & 7 \\
\hline hearing aids fitting & & & & & & & & & & 2 & 7 & 5 & 14 \\
\hline virtual reality & & & & & & & & 1 & 1 & & & & 2 \\
\hline database retrieval & & & & & & & & 2 & 1 & 8 & 8 & 1 & 20 \\
\hline knowledge acquisition \& data mining & & & & & & & 5 & 3 & 3 & 1 & 4 & & 16 \\
\hline image processing & & & & & & & & & 1 & 2 & & & 3 \\
\hline control \& robotics & & & & 1 & & & & 2 & & 3 & 4 & 4 & 14 \\
\hline internet & & & & & & & & & & 1 & 2 & 1 & 4 \\
\hline food industry & & & & & & & & 1 & 1 & & & & 2 \\
\hline geophysics & & & & & & & & & & & 1 & 2 & 3 \\
\hline art education & & & & & & & & & & & & 2 & $\overline{2}$ \\
\hline writing education & & & & & & & & & 1 & 3 & & & 4 \\
\hline games and therapy & & & & & & & & 1 & 1 & 1 & & & 3 \\
\hline social system & & & & & & & & & & 1 & & & 1 \\
\hline discrete fitness value input method & & & & & & & & $\overline{5}$ & & 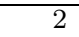 & & & $\overline{7}$ \\
\hline prediction of fitness values & & & & & & & 1 & 2 & 1 & 8 & 3 & 1 & 16 \\
\hline interface for dynamic tasks & & & & & & 1 & & & & & 1 & 3 & 5 \\
\hline acceleration of EC convergence & & & & & & & & 1 & 1 & 3 & 1 & & 7 \\
\hline combination of IEC and non-IEC & & & & & & & & 1 & 2 & & & & 3 \\
\hline active intervention & & & 1 & & & & & & & & 3 & 2 & 6 \\
\hline total & $\overline{2}$ & $\overline{0}$ & 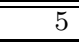 & 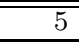 & 8 & 11 & 23 & 28 & 22 & 48 & $\overline{57}$ & 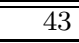 & 252 \\
\hline
\end{tabular}

\section{IEC Applichtions}

IEC research conducted during the 1990s originated from the work of Dawkins in 1986 [30]. Statistics on IEC papers are shown in Table I. In general, two major research streams developed during the 1990s.

One research stream is Artificial Life. Researchers and artists were interested in homegrown computer graphics (CG) and music according to an increasing interest in Artificial Life. The major IEC research during the early and mid-1990s were along this line, especially research on artistic image creation [12]. A practical application field, industrial design, forms a part of this stream.

The second research stream comes from the increase of researchers who are interested in humanized technology or human-related systems. These researchers have applied the IEC to engineering and other fields. A feature of this line of research is that the research on the IEC interface and human fatigue increased as applications developed. Recent IEC research has expanded into practical fields such as engineering or edutainment, unlike the early stages when IEC research was primarily biased toward artistic applications [203], [204], [206], [207], [213], [216].

The main objective of this paper is to provide an overview of all IEC-related research. This section surveys several IEC applications according to the artistic, engineering, and edutainment fields.

\section{A. Graphic Art and CG Animation}

The first IEC research was the biomorph of Dawkins, whose theory of evolution, selfish gene, upset the tranquility of the Society [30], [31]. He used an L-system that mathematically expressed a recursive development process of plants or other. He iterated two operations, which are subjective selection of the L-system outputs and mutation of genes that express the number and angles of L-system branches, and created several insect-like 2-D CG forms.

Many applications creating CG followed the biomorph; they were similar biomorph [190], plant CG based on the Lsystem [119], [145] (the main topic of the reference [145] was to avoid user interaction by making a fitness function for an aesthetic planned image although it handled IEC), 2-D CG based on mathematical equations or cellar automaton rules [1], [10], [11], [49], [50], [68], [112], [175], [187], [188], [189], [232], [238], [239], [240], [241], [242], [247], [251], 3-D CG rendering [231], animal CG [46], and airplane drawings with evolving wings and bodies [133], [134]. Mathematical equation-based IEC uses several methods to create CG: CG creation using nonlinear mathematical equations generated by GP, linear and nonlinear fractal transformation equations within the framework of an iterated function system, and given differential and dynamic equations. The parameters or structure of these equations are modified by IEC.

An artistic system, Mutator, has been applied to a number of animation applications, cartoon face drawing, commercial 3-D screen savers of Computer Artwork Ltd., mu- 
sic, and financial planning in addition to 2-D and 3-D CG [233].

An aquarium scheduled to open in Nagasaki, Japan in April 2001 will have a virtual aquarium as an annex. One of the virtual aquarium projects is to let visitors who have no CG experience create their own 3-D fish CG using IGA and release the fishes in the virtual reality space [70], [83]. The shape of the 3-D fish is expressed by mathematical equations and the parameters of the equations are modified by the IGA. The project also includes the development of an internet version of the IEC interface that allows visitors to create their own fish on the internet before visiting the aquarium.

The IEC was applied to generate 3-D shapes and texture that are expressed with Fourier transform in a virtual reality space [108]. The IGP was also used to create 3-D CG and music source localization in a CAVE for virtual reality although this research was primarily for virtual reality research rather than artistic creation [29], [32], [164].

One of most active IEC graphic artists would be Sims ${ }^{1}$. He used GP, evaluated CG created by the mathematical equation, evolved the mathematical equation by GP, and created fine $\mathrm{CG}$ art. The equations are used to calculate each pixel [187], [188] or create graphic movies by adding a time variable to the dynamic differential equations [188], [189]. He created several graphic art pieces including Panspermia and Primordial Dance in 1991 and 1993, respectively, and also lets visitors interact with his interactive art system at art shows and exhibitions. His Galapagos is an L-system-based IEC system that runs on 16 graphic workstations and a parallel machine, allowing visitor create their own graphic art through their interaction, and is exhibited at the multimedia museum, ICC (NTT InterCommunication Center), in Tokyo.

Next, we examine how to create IEC graphics using public-opened SBART ${ }^{2}$ [238], [239], [240], [241], [242] as an example of IEC art system ${ }^{3}$. The SBART creates tree structures of mathematical equations using GP and calculates the values for each pixel using the mathematical equation and the $(x, y)$ coordinate values of the pixel. The SBART assigns arithmetic operators such as the four fundamental operators of arithmetic, a power operator, $\sqrt{ }$, sin, cos, log, exp, min, and max to its non-terminated nodes and constant and variables (XY0 in Fig. 3 ) to its terminator nodes. Three values at each pixel are calculated using one generated mathematical equation by assuming that the constants are 3 -D vectors consisting of three real numbers and the variables are $3-\mathrm{D}$ variables consisting of $(x, y, 0)$. The three calculated values are regarded as members of a (hue, lightness, saturation) vector and are transformed to RGB values for each pixel. These three values are normalized into $[-1,1]$ using the following saw-like function:

$$
f(x)= \begin{cases}x-4 m & (4 m-1) \leq x \leq(4 m+1) \\ -x+4 m+2 & (4 m+1) \leq x \leq(4 m+3),\end{cases}
$$

${ }^{1}$ See his works at http://genarts.com/karl/.

${ }^{2}$ SBART is downloadable from http://www.intlab.soka.ac.jp/ unemi.

${ }^{3}$ The reference [176] introduces 14 IEC-based 2-D CG, 3-D CG, and music package with their URLs.

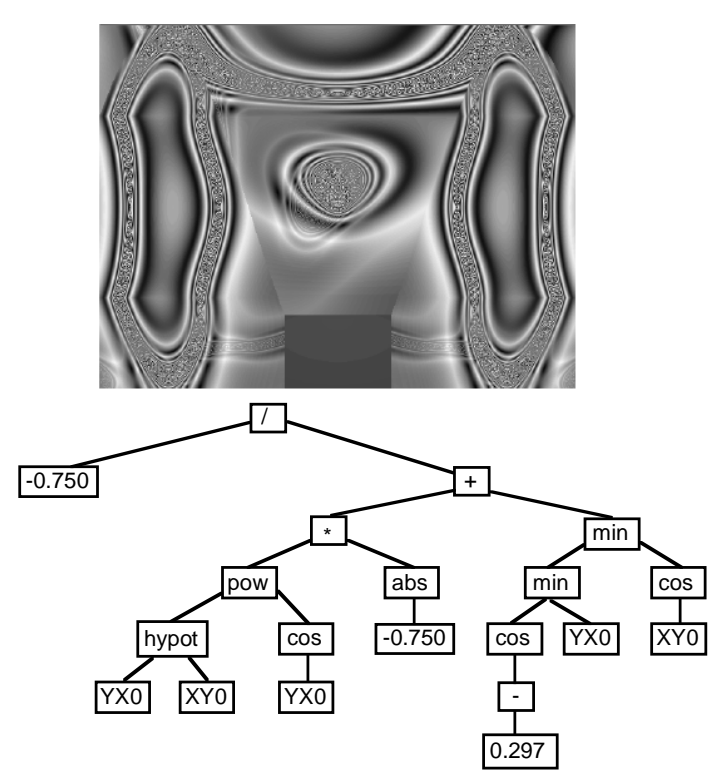

Fig. 3. SBART: simulated breeding-based 2-D CG system. GP evolves mathematical equation that is applied to each pixel, and graphics created by the equations are evolutionary obtained.

where $m$ is an integer. A graphic movie is created by replacing the constant $(x, y, 0)$ with $(x, y, t)$, where $t$ is a time variable. Also, the functions of the SBART were expanded to create a collage [241], [242]. A human user selects preferred 2-D images from 20 displayed images at each generation and IGP creates the next 20 offspring. Sometimes exporting/importing parent among multiple SBART user interface is allowed. This operation is iterated until the user obtains a satisfactory image.

An interesting EC operator was proposed and used to created deformed offspring images [47]. Selected parent bitmap images are lined up and regarded as time sequential signals, and each vertex or pixel point of offspring image is calculated from the time sequential points in time sequential parent images by applying an auto-regressive model.

Besides previously mentioned Panspermia, Primordial Dance, and SBART, Mutator [231] and some works [251] at International Interactive Genetic Movie, the third webbased exhibition, are graphic movies that add a time parameter to still image coordinates $(x, y)$. Fractal movies were created by the IEP using affine transformation that linearly maps a coordinate to another [1]. As the affine transformation creates only a still image, meta-level transformation that transforms a set of affine transformations to another fractal set of affine transformations was defined and used to create a graphic movie. The coefficient matrix of the affine transformation is renewed by the IEP, and a user rates 10 movies at each generation.

The IEC was used to create not only graphic movies but also animation of concrete objects. Comical movement of a deformed line body was created by the interaction between an animator and the EC [244], [245]; the walking motion trajectory of a linear body was created by finding a suitable combination of joint angles for the arms and 


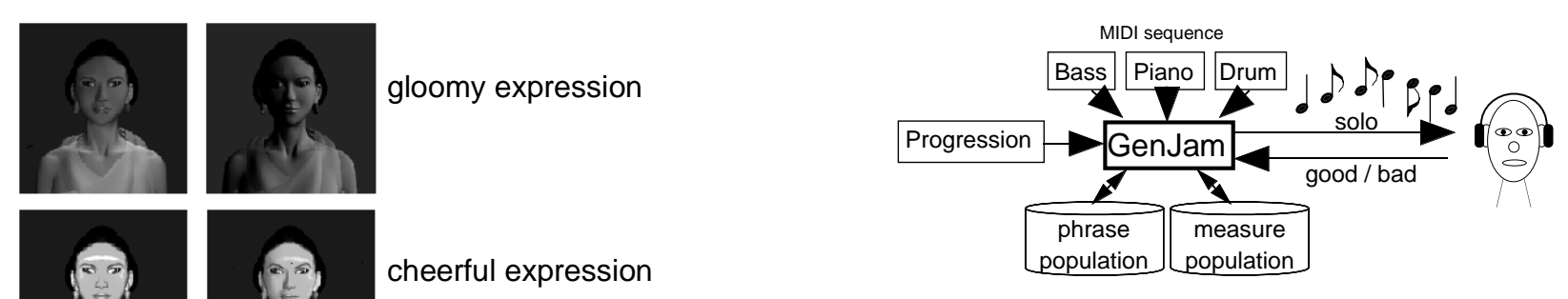

Fig. 5. GenJam system that interactively generates a jazz melody.

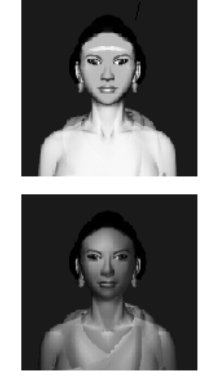

by hand

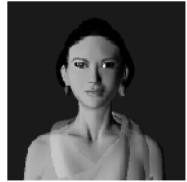

heroine expression

in a movie pamphlet

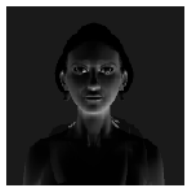

villain expression in a movie pamphlet

by IEC

Fig. 4. 3-D CG lighting arts whose design motif is "debut of a villain actress" made by an amateur with manual (left) and with IGA (right).

legs using the IEC [109]; animated pass-motions of two arms were generated by combining a multi-objective GA and IGA [184], [185].

\section{B. 3-D CG Lighting Design}

Since 3-D CG is a simulation of a photograph, CG impression is dramatically changed by lighting conditions such as positions, lighting, or colors of lights as well as photographs. Most of normal people can distinguish good CG lighting from bad CG lighting, nevertheless they do not have CG lighting skills to realize the intended impression. The role of lighting design support deeply influences CG quality and has gradually become gain importance as CG has spread from the professional to the business and hobbyist arenas. The IGA was applied to develop a 3-D CG lighting design support system [3], [5], [4], [6], [7].

IGA-based and manual-based CG lighting designs were compared. Experimental subjects were requested to adjust the impression of a CG woman by using three lights of two different types, an infinite one and omnidirectional one, sixteen levels of intensity, an on/off state, and the coordinates in a 3 -D space. Fig. 4 shows the comparison.

Subjective tests and statistical tests for the comparison showed that the IGA is not significantly useful for experienced $\mathrm{CG}$ designers but for $\mathrm{CG}$ designers with little or no experience. Experienced designers' explicit intentions shortens their manual lighting design times, while amateurs' designs, often are accomplished by trial and error, which takes more time than that of the IGA.

\section{Music}

IEC was applied to generate the melody [13], [16], [17], [18], [19], [20], [131], [132], [160], [243] and rhythm of percussion parts [56], [236], [237].

Sonomorphs is the first IEC-based music system, which was motivated by the biomorph of Dawkins as you see by its name, and it generates measure to phrase lengths of melodies based on a user selection [131], [132]. Sonomorphs runs with MAX, a popular object-oriented computer music language.

Fig. 5 is the IGA-based melody generator, GenJam, that inputs rhythm sections and chord progressions and generates a jazz melody. A user listens and evaluates the generated melody by tapping good or bad keys while the melody is played. Audio CD made by the GenJam was put on the market [14]; the performance that the majority decision of the audience was fed-back to the GenJam to generate jazz music [15]; and performers can play with the GenJam in real-time [17], [18]. Another IEC-based music generator has an IEC interface as same as an IEC-based image generator [243].

It takes too much time and causes too much human fatigue if a user gives one fitness value after each melody that is played. The GenJam allows a user to evaluate the generated melody measure by measure instead of entirely melody by melody to avoid this problem [13]. This method shortens IEC time is useful for other IEC tasks that handle time-sequential signals.

Vox Populi is a composition system that allows a user to change the parameters of a fitness function in real time instead of giving subjective fitness values [123]. A fitness function that evaluates how each melody from soprano to bass satisfies each voice part range and harmony degree is set in the system. A user listens to the melody generated by GA and controls the parameters of the fitness function through five user interfaces.

Although it is not music, the IEC was applied to control the parameters of FM sound synthesizer to realize a mental tone [69].

\section{Editorial Design}

The IEC was applied to design HTML style sheets [121], [221]. As the internet advances and expands from businesses to homes, the number of people who open their private web page as hobby is increasing. Many visual HTML editors are found in the commercial market and at free \& shareware software sites, and designing homepage is now 
taught at many elementary and junior high schools. The impression of a web page depends on a combination of background color, font type, size, and color, font of title level, link color, and so on. The IEC is a good tool to visually optimize this combination.

The IEC was applied to design a poster by optimizing the combination of its layout, font size, and color [120], [144]. A user expresses the impression of a poster with the degrees of 12 impression words after he or she inputs sentences specifying image file names to be used in the poster. Fuzzy logic rules describing layout knowledge of experts in their system estimate the impressions of posters generated by the GA with the degrees of twelve impression words. The distance between user's and system's impression degree vectors was used as a fitness value, and offspring poster layouts were generated [120]. Same design is conducted the font and color for a color poster design as well [144]. Their fuzzy logic system has a learning function to absorb the personal difference of impression. The HTML style sheet color design of [221] is based on this system.

As well as systems described in section III-J, it is best if the optimal poster is obtained at once, otherwise the user continues to iterate the EC search by changing the coordinate of target impression on a psychological space, which corresponds the EC search in next generation.

\section{E. Industrial Design}

Most industrial projects are designed using CAD systems. It is easy to understand that industrial design is a good application task of IEC because it is a form of design parameters such as lengths, angles, coordinates, color numbers, and so on. Several IEC applications for industrial design include car design [44], [45], [76], fashion design [28], [78], [79], [80], [81], [82], [128], [129], [130], color design for knitwear or graphics [34], and shape development for new tools, such as scissors, saws, and any other general 3-D object shapes [91], [92], [178], [179], [183]. There is a formation based 3-D form design approach that encodes five transformation commands of insert, delete, fold, lift, and pole-hole as genotype code and interactively creates new 3-D forms [143].

The IEC was also applied to civil engineering and architectural design. The designs of concrete arch dam [165], suspension bridges [40], houses [45], [48] are some of its applications. Graf et al.'s system displays bitmap images generated by warping and morphing operators that interpolate the vertices and points on two parent bitmap images onto an offspring bitmap image [44], [45], [48]. As they use photo images as initial EC parents, their car or architecture design has reality. This system seems to be widely useful for general design in industrial, architecture and civil engineering designs.

Positional design of HyperCard on a screen and general layout design being applicable to GUI design [58], [116], [117], [118] and floor planning [35], [191] can be categorized in this section. Masui's layout design makes a fitness function adapt to a user's preference by teaching GP which layouts are good or bad [118].
The IEC was applied to the layout of the interior furniture. The furniture layout may depend on personal preference, functional relations of furniture positions, the purpose of the room, or even Feng Shui, frequently used in Chinese society. An IGA-based interior layout system where the GA optimizes the positions of furniture with fitness values consisting of objective evaluation based on expert knowledge and subjective evaluation based on user's preference was presented [89].

\section{F. Face Image Generation}

Montage systems are the systems that combine partial images of facial photos and compose a facial image. The montage system used at Fukuoka Prefectural Police in the author's resident area combines the partial images of 50 faces of people to compose a criminal suspect's face. Clear memory of partial facial features and explicit indication of partial facial images are necessary for a conventional montage systems, but it is very hard for a general witnesses to memorize a suspect's face in detail. IEC-based montage systems optimize the combination of partial facial images and generate a facial image based on the witness's total impression of a suspect in mind rather than the memory of each facial part [25], [84], [85], [211]. While the montage systems uses 2-D photos as original images, 3-D facial expression whose data are obtained by a $3-\mathrm{D}$ digitizer was created using the IGA [67].

Generating faces of line drawing was frequently used as a research task of the IEC [8], [9], [57], [126], [127], [135], [136], [192], [193], [194]. One such research is the autofitness assignment to accelerate EC convergence by estimating fitness values using the Euclidian distance from individuals [126], [127] and the positional relationship with user-selected individuals [57], [135], [136], [192], [193], [194] (c.f. section IV-B).

The expression of facial photo images can be changed by deformation that the pixel positions of the facial parts such as the eyes, the eyebrows, or the mouth area are continuously mapped to other pixel positions. The IEC was used to generate this facial expression [107].

\section{G. Speech Processing and Prosodic Control}

The IEC was used to design a digital filter that audibly reduces speech distortion. Concretely, the GA optimizes eight coefficients of FIR distortion reduction filters [249], [250] or amplification levels of each frequency band [234], [235] for distorted input speech whose power in low frequency range where the voice formants exit is suppressed, and a user hears the recovered speech, evaluating its quality, and feeding back his or her subjective fitness.

The result of the subjective tests showed that the quality of the recovered speech was significantly better than original distorted speech not only for the IGA users but also for other subjects as well [249], [250].

The IEC can be also applied for speech synthesis. Speech has phonetic and prosodic information, and voice impression and naturalness are controlled by prosodic parameters of pitch, amplitude, duration, and speed. Linguists have 
tried to make rules of the relationship between the impression or naturalness and the prosodic parameters in conventional speech synthesis research. Prosodic control is a good task for the IEC because the quality or naturalness of synthesized speech can only be evaluated by humans. The IEC was used to modify prosodic parameters to change voice impressions to reflect five impressions such as peace, anger, or joy [180], [181], [182].

Microsoft Agent is a character agent whose behavior and prosodic parameters can be controlled by user's program. The IEC was applied to select one of 40 prepared its behaviors and the control pitch, amplitude, speed, and emphasis parameters of its voice to realize a human-like emotion of the agent [122].

\section{H. Hearing Aid Fitting}

Anticipating the onset of an aging society, digital hearing aids using digital signal processing have spread and solved several problems that conventional analog type of hearing aids cannot overcome. However, hearing aid fitting that maximizes the performance of hearing aids to a wide variety of users has not yet established as well as natural sounding quality. The essential reason is that only the user can evaluate the hearing quality; no one can perceive how another person hears.

The IEC has potential for automatically tuning the parameters of the signal processing according to the user's hearing perception, so that IEC Fitting was developed (see Fig. 6 ) [146], [151], [208], [209], [217]. This approach is essentially different from those of conventional hearing aid fitting and auto-fitting, and it tunes the hearing aid parameters based on how each of hearing impaired persons hears without previously measured their hearing characteristics. So far, hearing aid engineers or audiologists measure the hearing characteristics, such as with an audiogram, and adjust the hearing aid parameters to compensate the difference of the characteristics between the impaired person and normal hearing persons. The problems of this conventional approach are: sound source for measurement is limited only pure tone or band pass noise, measurable hearing characteristics are just few parts of a human auditory system, full measurement takes long time, previous measurement of the basic hearing characteristics. Thanks to its sound source-free characteristics, the IEC Fitting was evaluated with speech, speech with multi-conversational noise, and music and shown that its users' satisfaction and intelligibility were higher than those of conventional fitting [152], [153], [154], [156], [209], [212]. Comparison of two fitting methods has also analyzed [38], [39].

An IEC Fitting system for one type of a hearing aid on the market was constructed for a potable PC and is on field test with actual users [39], [155], [248].

\section{Virtual Reality}

The IEC may solve the problem of which factor gives us virtual reality (VR). Imagine the VR control of an arm wrestling robot fighting against a human (see a photo in Fig. 7). Whether a human participant feels VR in the

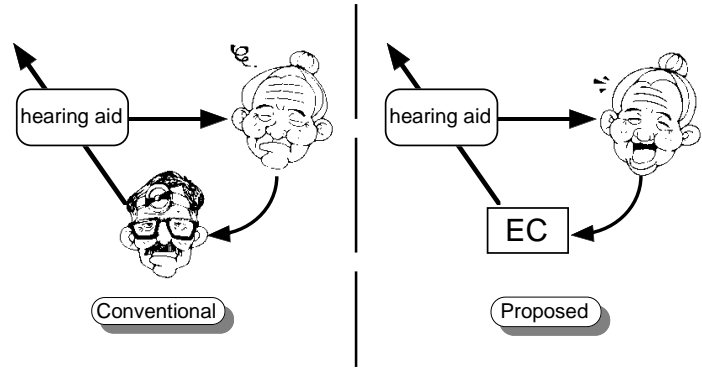

Fig. 6. Conventional hearing aid fitting (left) and IEC Fitting (right).

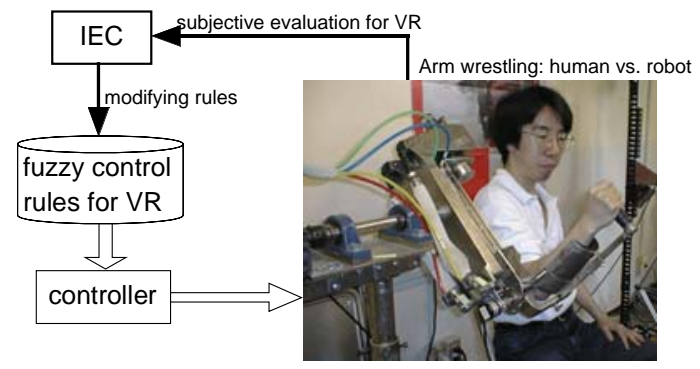

Fig. 7. VR of arm wrestling. Fuzzy logic controller evolved based on human perception of VR.

action of the arm wrestling robot or mechanical swinging depends on the control rules.

We obtained the control rules to win using a classifier system in the first stage. When the arm wrestling robot pushes a human participant forward, the control rule of the classifier system is awarded, and 20,000 rules were obtained. Then, the 20,000 classifier rules were complied into 8 fuzzy rules to help our analysis. This compilation was conducted by using fuzzy knowledge acquisition techniques based on the GA [72], [73].

The final stage is that the IEC modifies the fuzzy control rules for winning to those for VR. Since only a human participant can evaluate how he or she feels as if the robot were a human, we can use the IEC to modify the parameters of the fuzzy control rules. It is expected that the VR factors of the force perception may be explicated by analyzing the difference between fuzzy control rules for winning and those for VR.

\section{J. Database Retrieval}

Suppose that we wish to retrieve an image or music from a huge database or from the internet. In most cases, especially for the case of images, the media that we want to retrieve is not a specific one but one that is suitable for a certain purpose or that is preferable. Retrieval with keywords can rarely be used for this purpose, and we often do not know what image or music is in the huge database or on the internet.

The IEC is applicable to content-based media retrieval and can find out image, music, or other media that match a certain purpose or user's preference based on human evaluation of the retrieved ones.

The EC searches media on their feature paremeter space, 


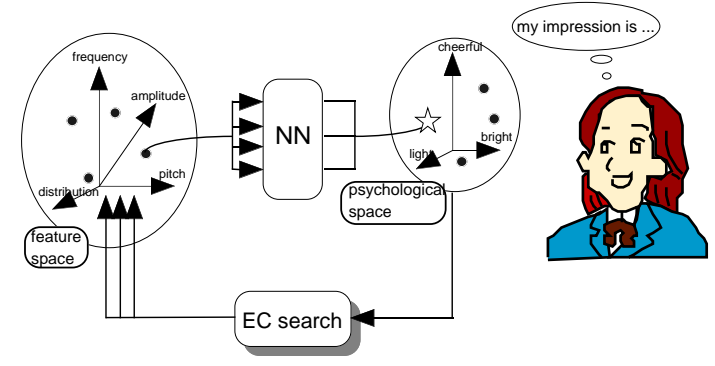

Fig. 8. Media database can be retrieved by reverse-mapping from the psychological space of a user to the feature parameter space of the media in the database after obtaining the opposite mapping relation.

while human evaluates them on a psychological one [26], [27], [28], [100], [101], [102], [103], [186] such as Fig. 2 or an artificially constructed psychological one [140], [141], [210], [214] for intelligibility. A mapping method from the psychological space of the latter to the feature one is needed to find the best matched media to the target point in the psychological space. However, the dimension number of the psychological space is generally so far less than that of the feature paremeter space that it is difficult to map in this direction. Conversely, the mapping in the opposite direction is easier. Neural networks (NN), fuzzy reasoning, or other nonlinear mapping methods are suitable to map from $m$-D space to $n$-D one where $(m \geq n)$. Once a nonlinear mapping system learns the mapping relation, we may be able to use the trained nonlinear mapping system for this direction, and GA was used for the inverse mapping (see Fig. 8).

Sometimes a user must feel that the retrieved media does not match the target impression. There are two user choices in this case: to start again at the beginning or to try changing the target coordinate in the psychological space. The IEC is used for the latter.

Several papers applied the IEC to image database retrieval. Some of them are: interactive image browsing using pipeline-type GA mentioned later [86], [87], [88], interactive image retrieval using wavelet image feature [26], [27], [28], [100], [101], [102], [103], image retrieval that uses color, texture, position of meshed image areas as features and that assigns bias fitness values to images that are not displayed while the user rates the displayed images [186], image material retrieval for multi-media title design [125], and national flag retrieval [115].

The image material retrieval system for multi-media title design uses the IEC to determine the user's preferred images and time-sequentially orders them. This system uses Messy GA to increase the redundancy of gene codes, which results in reducing the load of interactive user-selection [125].

The idea of a media converter was proposed. It combined IEC-based image and MIDI file retrieval systems and unified their psychological spaces, which can realize the retrieval of image features-psychological space-music features direction and its opposite direction [142], [214].

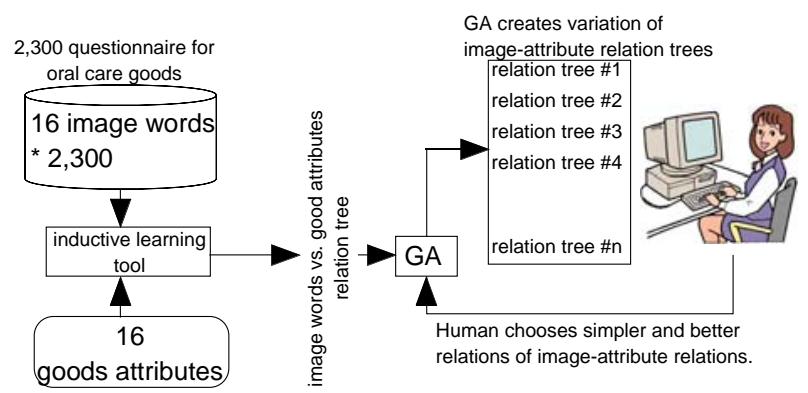

Fig. 9. Reliable rules with fewer number of attributes that characterize each oral care product are determined from questionnaire data using the IEC and a marketing expert.

Therefore, computers can show us an image of same impression when we input the music, and vice versa.

As the EC usually searches a feature paremeter space for the spatial distribution information such as color and gray levels, it is impossible to retrieve media at a semantic level. For example, it is hard to retrieve an image of a smiling girl in the dark with happy impression. If the semantic level is limited to basic shapes such as triangles, squares, or circles, they may be able to be used as image features for image retrieval at the semantic level. There was an IEC-based image retrieval that handled the semantic by limiting the retrieved target to only simple images [74], [75].

\section{K. Knowledge Acquisition and Data Mining}

When a new version of product is developed, the customer target and product concept are first determined according to market research, and several attribute parameters of the product are adjusted to match to the new concept. The problem is matching the parameters. Their relationship rules may be obtained from past market research, but noise in the questionnaire survey from consumers is inevitable.

The IEC was applied to acquire marketing knowledge of oral care products from the questionnaire data [63], [64], [222], [223], [224], [225], [226], [227], [228], [229]. Their approach consisted of: (1) basically applying inductive learning to obtain the relationship rules from the data, (2) increasing diversity of possible attribute parameters in the rules using genetic operations, (3) using expert choices of better rules through the IEC process, and (4) finally determining a reliable decision making rules or trees with fewer number of attributes that characterize each oral care product (see Fig. 9).

The inductive learning and the IEC with an expert are applied to 2,300 questionnaires with 16 image questions such as "better taste and smell" and "usable for all family." The study revealed that the obtained rules have a dramatically small number of product attributes and small size of rules, and the obtained rules show the marketing strategy for a certain company. 
The same approach was applied to the questionnaire data for a beer product [65], [66] and clinical data [59], [230]. The rules obtained by inductive learning were evaluated by the experts of marketing or clinical medicine and were polished up by a simulated breeding.

The IEC was applied to another type of data mining based on 2-D mapping. GP generates nonlinear functions that inputs all attribute parameters of target data and outputs two coordinate values, $\left(v_{1}, v_{2}\right)$, i.e. this function maps data from an $m$-D to $2-\mathrm{D}$ space. The multiple data distributions on the 2-D space generated by the GP are displayed to a user, and the user selects visually meaningful distributions of the data. The point of this research is the meaningful; the meaningful distribution implies that the mapping function generated by the GP may have some kind of meaningful knowledge. For example, separated four clusters for four-class data looks meaningful than randomly mixed distribution. The selected ones evolve, and the offspring distribution maps are generated. It is reported that this method acquits proper knowledge [246].

A combination of normal GP and the IGP was applied to obtain knowledge that classified eight kinds of damage data of stainless steel [196]. Unlike the normal IEC, human interaction was not conducted for every generation but for every 100th generation to speed up the IGP. The GP generates rules classifying the types of damage by inputting 17 parameters of damaged data in the experiment. It was reported that fitness values were drastically changed at the 100 th or 200th generation and the rules obtained by the GP showed robustness for noisy data.

\section{Image Processing}

Image enhancement for medical images is helpful and necessary for medical doctors to detect diseased parts easily and correctly. The performance of the image filters for medical image enhancement is evaluated by only humans, particularly medical doctors, and the best enhanced image might depend on the doctors' visual preference. This is a good IEC task.

The IGP was applied to design image filters that enhance MRI (magnetic resonance image) and echo-cardiographic images [174]. The GP creates mathematical equations that describe the image filters based on the ability of human visual distinction.

The IEC was applied to detect the outline of plants in an image. Image processing that segments plant parts and detects their outlines is necessary to obtain the growth information such as shape, growth rate, or leaf color from tele-monitored plant images. To obtain the best image processing filters, the GA optimizes their coefficients to best match a filtered image with the objective plant image that a user traces on an original image using a drawing user interface. The combination of a fitness function showing the matching degree at each pixel and human visual evaluation is fed-back to the GA [161].

Another IEC approach is to determine the sequence of image filtering. As the popularity of digital cameras, image scanners, PCs, and the internet increase, the possibility that amateurs work with images also increases. Most amateurs use retouching software that prepares several image filters. Generally, different orders of image filtering results in differently retouched images, i.e. an image filtered by A, $\mathrm{B}$, and $\mathrm{C}$ is different from an image filtered by $\mathrm{C}, \mathrm{B}$, and A. Although it may be difficult for amateurs to decide on a filtering sequence, it is not difficult for them to evaluate which image is better or preferable. Simulated breeding was used to auto-generate the sequence of image filtering [124].

\section{Control and Robotics}

The IEC applications to control have recently increased in addition to the arm wrestling robot described in section III-I.

The first engineering application of the IEC was the GA control of a six-legged insect-style robot in 1992 [106]. Each leg had two neurons for swing and elevation respectively, and the leg movement was determined by the oscillation between the two neurons. Then, the locomotion of the robot was determined by the connection parameters among oscillators of the six legs. The GA optimized the first oscillator neurons based on human observation of each leg, i.e. IGA, and the latter control among legs was evolved based on a fitness function of how long the robot goes forward or backward with less totter.

A similar approach to NN control of a eight-legged robot introduced the IEC and three devices and dramatically decreased the number of GP generations from one million of their simulation to 200 of their hard system [51]. Another IEC approach was the obstacle avoidance control of a Khepera miniature robot [33]. The interaction between the GA and user's visual evaluation was used in their first evolving phase, but the interaction between the GA and a human evaluation model obtained by the observation of the user evaluation in the first phase was used in their second evolving phase to reduce human evaluation fatigue (see section IV-B).

The IEC was used to control a Lego robot to realize an interesting locomotion that children prefer. The connection weighs of the NN that inputs robot sensor information and outputs locomotion control values are evolved according to the selection of better locomotion robots by children [113], [114], [162]. The IEC realized a programming-less program that is best used for children's games and toys. It is surprising to know that only population size of nine moving robots generated the control NNs that realized the interesting movement of robots after only five to seven generations in the experiment [114].

The IEC was applied to generate humanity movements of robots. One application was planning a robot arm path so as not to frighten a human when a human and a robot do cooperative work such as handling goods [93], [94]. While the evaluation measure for planning the arm path of industrial robots is efficiency, such as the shortest distance or time, the minimum energy, or the most stability, a different measure is needed for that of consumer robots. Humans tend to fear an approaching lump of iron, robot arm. The 
objective of this research was to determine the best tracking path and speed of the robot arm that minimized the frightening feeling by using IEC. This type of approach became important for care robots, pet robots, or other consumer robots that human-friendliness is required rather than efficiency.

We can often guess human emotion from the body posture. It is possible to express such emotion in the movement of autonomous robots. This trial was conducted to optimize the consequent values of fuzzy control rules for robotic movement using the IES. A user observed the different movements of autonomous robots and chose the movements that seemingly mimicked emotions for which the user was looking, such as the emotions of happiness, anger, or sadness [77].

The IGA was applied to teach a pet type of robot new tricks. Human user observes the fuzzy-logic controlled movement of the robot on a 2-D plane, which means tricks here, and evaluates them by touching the body sensors of comfort and discomfort [95]. The evaluation is fed back to the GA to generate offspring tricks.

The IEC is also considered to control an NN controller that inputs the throttle angle and vehicle speed and outputs the air-fuel ratio to account for the user's preference of riding comfort [71]. As the first step of their research, they did not use the IEC for their simulation and an experiment with a real engine but used an EC and a human model of the NN that learned the relationship between machine data and user's preference instead of a real user. They are planning to use the IEC in future research [71].

When we design or acquire control rules, sometimes their intelligibility to explain them to other people has higher priority rather than the best performance. The IEC was applied to obtain such fuzzy control rules for car parking with the combination of a fitness function for objective evaluation of the control performance and human evaluation for the intelligibility of the obtained rules [2], [158], [159]. This research might be categorized in section III-K.

It was proposed to allow a user to directly indicate which control rules are better to increase the fitness during a normal fuzzy classifier system runs. Their trial to acquire fuzzy logic rules that control a robot to take a flag and return without colliding with other robots failed in their first simulation. Then, they observed the partially successful rules to reach to the flag in the 21st generation and indicated their classifier system to search in the neighborhood for the fired fuzzy control rules. This human intervention resulted in determining the successful robot control rules at the 23rd generation [37].

A recent topic on robotics in Japan is the spread of robots from industry to the home. The size of Honada's AIMO is determined taking account of the cooperation with a human at home. Five thousand units of the first model of Sony's AIBO were sold out within only 17 minutes through the internet in spite of an extremely expensive price of 250,000 YEN. This emphasized the entertainment aspect of AIBO as a pet robot. Seal and cat robots with fur, from the Mechanical Engineering Laboratory, or MITI, ap- peal to users' sense of cuteness or mental healing. The IEC technique will be in much demand for generating cute, natural, and fluid motions for consumer robots whose features do not necessarily include working efficiency but cuteness or safety.

\section{N. Internet}

As the use of the internet has dramatically increased, IEC has also been applied to disciplines related to the internet. IEC-based web page design [121], [221] described in section III-D is one such application. The features of the virtual aquarium project described in section III-A and the 3-D modeling education project described in section III-Q are design and education through the internet.

E-business is a topical keyword in this field, and IEC was applied to auto-design web banners [41], [42]. Like previously described artistic applications, the basic approach combines designing the parts and parameters by selecting parent banners according to user evaluation and EC operations. The biggest difference from other IEC-based arts is that the IEC users are not banner designers but customers. The banners are automatically created according to the number of customers' visits, i.e. the number of times the users click banners. This application does not contribute to the biggest IEC problem, the user fatigue problem, nevertheless the application can embed the customers' preferences and automatically design more attractive banners.

Another recent topic is the agent. Personified agents that advise users how to operate software rather than the use of on-line manuals has been recently put on the market. The Microsoft Agent is a character agent whose body behavior and voice prosody are controlled by a user program. The IEC was applied to reflect human emotion in its behavior and voice by selecting one of 40 prepared kinds of behavior and voice parameters such as pitch, amplitude, speed, and stress based on the user's subjective evaluation [122].

\section{O. Food Industry}

The IES was applied to coffee blending. Before the application, the performance of the IES was evaluated with three toy tasks [54]: (1) to determine the mixture ratio of three liquid primary colors of cyan-blue, magenta-red, and yellow and with clean water, which results the color of Cherry Brandy, (2) to generate a square whose size and color are same with those of the target square by optimizing the five parameters of length, width, red, green, and blue, (3) to generate a polygon whose shape is specified by line segments that radiate with equal angles from the origin coordinate and match to the target polygon.

Then, the IES was applied to coffee blending with the cooperation of three professional coffee tasters from a coffee roasting company in Berlin. The task was to determine the mixture ratio of five kinds of coffee beans whose coffee tastes similar to that of the target coffee. The IES determined the ratio that the professional coffee tasters could not distinguish [55]. 


\section{P. Geophysics}

The IEC has been applied to geophysics. The mantle convection has already been modeled with physical characteristics such as earth revolution. If this mathematical model is correct and the correct parameters of the initial earth inside situation are inputted into the model, the current underground situation can be estimated. However, since no one can know the past situation of earth inside for certain, the modification of the initial situation parameters and model inputs were iterated by trial and error according to the observation of simulated results. This task was categorized to a reverse problem because the model input was estimated from the model output. If this model simulation goes well, we may estimate where there are certain mines.

Expert knowledge of geophysics and geology and measured geophysical data are indispensable to the appropriateness of this simulation. So far, the initial values of the conventional simulation were determined by trial and error although the experts evaluated the simulation result. The IGA has been applied to this point; the GA determines the initial parameters of the simulation, and the expert evaluates the validity of the simulation results [21], [22], [24].

This approach was used to estimate the existence of heavyweight mass. When gravity from A point to B point on the earth's surface is measured, a non-uniform gravity graph is obtained when there is heavy rock or mines underground. The task is to estimate the depth, gravity, and size of the rock or mines. Although it is mathematically impossible to determine unique values of the three variables from the 1-D measured gravity data, the geophysical knowledge and experience would help to narrow the solution candidates down. Here, the IEC with a geophysicist is applied to estimate the combination of the depth of its location, gravity, and size of the rock [23].

\section{Q. Art Education}

Both an artistic sense and skill are essential for creating art. Artists are often trained to sketch and sculpt. However, artistic skill requires time to be cultivated similar to that of playing musical instruments. We can separate these two types of training and focus on education that perfects an artistic sense by introducing the IEC.

An IEC-based educational system consisting of a 3-D CG modeling model and the IEC changing the model parameters to create several $3-\mathrm{D}$ shapes to a given motif is being developed [137], [138], [139]. This system is directed toward education, especially developing an artistic sense rather than an artistic skill such as sketching and sculpting. This allows relatively unskilled art students to polish their artistic sense while their artistic skills are being perfected through training by the traditional practices previously mentioned. Fig. 10 shows an experimental scene of creating 3-D green peppers on a large screen by viewing the image from several angles. Now this system can be installed on portable PCs and can be brought to any class or school of artistic education. We are going to connect the internet version of the IEC interface and realize a remote education system for developing an artistic sense.

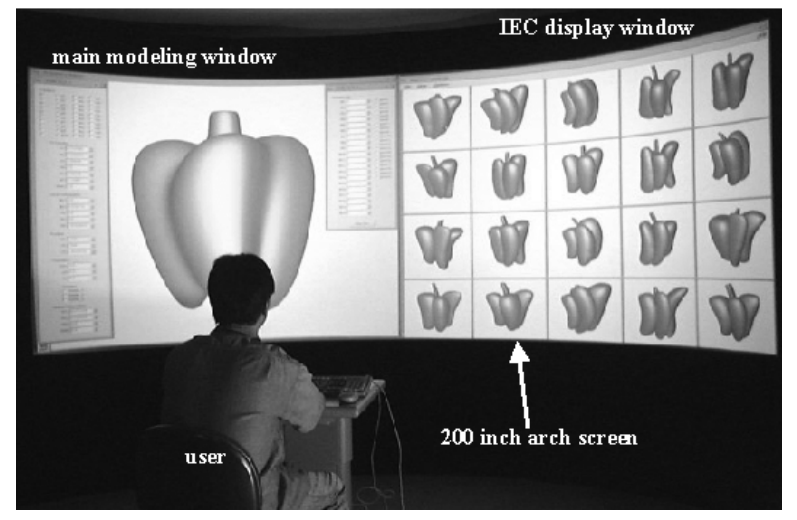

Fig. 10. IEC-based 3-D CG modeling system for art education.

\section{R. Writing Education}

Since the IEC displays several individuals, sometimes they give users hints or inspiration; it implies that the IEC is useful to stimulate human creativity. This inspired creativity obtained during the IEC iteration is more important for education than what the EC outputs, such as images or music.

This characteristic of the IEC was applied to develop a writing support system for children [96], [97], [98], [99]. Composing a story is considered more important and more difficult than writing sentences, and children in the lower classes are frequently puzzled. Their system displays 24 pictures and lets a child choose two sets of a four-picture sequence; this sequence establishes an initial storyline. Their system then creates several four-picture sequences using the selected parents' four-picture sequences and GA operations. The child then chooses two better four-picture sequences in the next generation. This iteration is repeated until a satisfactory story can be written.

\section{S. Games and Therapy}

Similar to educational applications, edutainment or games are a good application field of the IEC, because it is much easier for children to select better ones than give detail instruction or write programs. This selection corresponds to an award for reinforcement learning, and the IEC can train the control rules or mechanism of a target system similar to reinforcement learning.

Besides the Lego robot previously described in section III-M, some of IEC-based games are based on an artificial life survival game, painting graphics based on NN evolution, Artificial Painter [163], [247], and drawing faces [162].

It is reported that the drawing face software is used for not only edutainment but also mental therapy. Italian therapists joined and started a project to apply the software to encourage mentally diseased children to understand facial expression [162].

\section{T. Social Systems}

New types of IEC were proposed for social systems in which all EC operations were conducted by human evalu- 
ators. Since EC was a computational model of biological evolution, EC researchers naturally designed ECs for computers. Even the IEC applications described in the previous sections passed only specified parameters from the computers to the human evaluators. These new types of IEC were applied to two social systems.

The first system, Free Knowledge Exchange, is a forum on web to obtain valuable ideas, and its process is based on the IGA [90]. Text-based questions and answers (chromosomes) of words from a natural language (genes) compose the knowledge database (population). Forum participants review a combination of questions and answers and provide their own answers or ideas (crossover). Participants can alternatively be inspired by previously submitted answers and propose an entirely new answer or idea (mutation). Each answer is then evaluated based on the number of participants interested in a particular answer or idea (human evaluation of fitness). New answers or ideas (offsprings) are generated by this process.

The second system, Teamwork for the Quality Education, a course proposed during the curriculum reformation of engineering education, is based on team competition in academics, service and design, and summer-job placement. The course was tested during the Spring and Fall semesters of 1997 in the General Engineering Department at the University of Illinois [43]. Although the original reference [43] did not explicitly describe the relationship between the project rules and the GA process, the reference [90] indicated that teams (chromosomes) of students (genes) competed for a team evaluation (human evaluation of fitness) during each semester (generation). Team members were first assembled by a draft (initialization), and the team competition (selection) was conducted by swapping team members (crossover) and by imitating other team activity (mutation) at the start of the next semester.

\section{U. IEC of the Broad Definition}

We are focusing the IEC researches of the narrow definition in this paper (see section I), but let's see some of those of the broad definition in this section. To distinguish both definitions of the IEC, we temporally use the terms of narrow-IEC and broad-IEC only in this section.

Most broad-IEC research would be multi-objective optimization research introducing the GA. Most of multiobjective optimization papers whose titles include the term of interactive are included in the broad-IEC research. Some of GA-based multi-objective optimization, such as the research of multi-objective evaluation consisting of user's subjective evaluation in part [220] and that letting a user subjectively give weights to multiple fitness values [195], should be categorized in the narrow-IEC.

The broad-IEC was applied to a nurse scheduling support system [62] that might be in the narrow-IEC. Although the GA makes a schedule for nurses based on the given fitness function, sometimes a head nurse may find it inconvenient or unacceptable to schedule results because it is difficult to design the fitness function to fully satisfy the user's specifications. In this case, new constraints of nurse scheduling that the head nurse finds are added to the fitness function, and the GA search is restarted. This is, this system optimizes the scheduling based on the interaction of a human and GA, but subjective evaluation is not directly used as a GA fitness value but used to correct the fitness function.

Computational biology is a new approach in pharmaceutical industry, and the GA is used for a simulation to generate a new molecular system by combining biomolecules. The GA searches the most tight bond of the biomolecules, i.e. the combination that minimizes the free energy of the obtained molecular system, by changing the combination of six parameters such as rotation of the molecular in a $(x, y, z)$ space. After several GA generations, a human generates a new individual molecular system by manually combining two biomolecules in a virtual reality space, CAVE, and adds it as a new elite [105]. Although this is not a narrow-IEC application, it is an interesting GA-based human-machine interaction system in a new field.

The GA takes a longer time to solve a TSP (traveling salesman problem) according to its size. There is a proposal to allow a user to interactively divide a big TSP into smaller TSPs and visually combine the solutions of the smaller TSPs to obtain the solution of the final larger problem TSP to reduce GA calculation time [111].

The interactive evolutionary design environment that Parmee et al. developed with British Aerospace is a design system through the interaction of a design team and the system with a built-in GA [166], [167], [168], [169], [170], [171], [172], [173].

\section{Non-Applicational Researches}

The biggest remaining problem of the IEC is reducing human fatigue. Since a human user cooperates with a tireless computer and evaluates EC individuals, the IEC process cannot be continued after many generations, prohibiting the practical use of the IEC. The major part of the following second and third problems are directly related with this first problem.

The second problem is how to search for a goal with a smaller population size within a fewer number of searching generations. The IEC has to search under this condition due to the limitation of the individuals simultaneously displayed on a monitor, the limitation of the human capacity to memorize the time-sequentially displayed individuals, and the requirement to minimize human fatigue. Since this limitation results in poor and slower EC convergence, we need to develop methods that EC converges under this condition.

The third problem is how to let an IEC user evaluate time-variant individuals such as sounds or movies with less fatigue and less operation time. The IEC has to timesequentially display the time-variant individuals. Since the IEC user is forced to compare the current displayed sounds or movies with previous ones in the user's memory and to evaluate them, the user's psychological fatigue increases and total operation time becomes long. The same situation sometimes occurs even if the outputs are spatially displayed 
still images. If the images are detailed or large, they should be either displayed individually or a few images at time. If the population size is increased to solve the second problem, the individual images must be displayed in sets due to the maximum number of images simultaneously displayed on a monitor.

The good news is that many IEC tasks do not require a large number of generations to achieve satisfactory results. Due to a good initial convergence of EC, unlike gradient methods, the human fatigue problem may be less tiring than from gradient searches. Task characteristics of subjective searches and numerical/combinational optimization are quite different, and the former does not have the exact optimum point. This is why it is sufficient for the human evaluated task to reach to an optimum area rather than a single point, which searches a satisfactory solution with fewer searching generations.

Nevertheless, we must solve the fatigue problem to make the IEC practical. Most non-application research are related to the IEC interface research in order to solve this fatigue problem.

\section{A. Discrete Fitness Value Input Method}

Psychological fatigue is deeply influenced by the ease of evaluating the outputs of the IEC and of providing feedback on the evaluation values to the EC. For example, as we cannot exactly distinguish the difference between 62 and 63 points in a rating of 100 levels, to determine 62 or 63 points in our subjective evaluation to individuals results in psychological fatigue.

It is expected that users can daringly evaluate the EC individuals and therefore reduce their psychological fatigue with broad ratings, such as five or seven rating levels, rather than a higher order of level ratings. Such a psychological discrete input method that distinguishes from 100 or 200 levels rating is proposed [147], [197], [198], [199], [200], [201], [205]. Since the rougher level rating results higher level of quantization noise, the EC convergence may become worse. We evaluated the total performance of the proposed method by taking into account both the advantages and disadvantages.

The subjective test and statistical test showed that the proposed method significantly reduces human fatigue. A simulation experiment showed that the worse convergence becomes significant when the EC search reaches several 10s or 100s of generations. It showed that the poorer convergence in practical IEC generations, such as fewer than the first 10 or 20 generations, is not problem. This simulation result supports the result of the subjective test [147], [198], [201].

\section{B. Prediction of Fitness Values}

Some methods that predict the fitness values of individuals for fast IEC convergence as a solution to the second IEC problem previously described were proposed. If the IEC has a predictive function, it can increase the searching capability by using a large population size equal to that of a normal EC and not increase the user's fatigue by display- ing only a few predicted individuals that have higher fitness values. Two prediction methods that learn user evaluation characteristics and predict fitness values were proposed; the first method uses distances in an EC searching space, and the other uses an NN.

One typical distance-based approach prepares a function that predicts the fitness values of any individuals based on the Euclidian distance from the individuals that a user gives as one's subjective fitness values, to apply the function to 200 individuals, and to display only the best 10 of the 200 individuals to the user for subjective evaluation [126], [127]. One improvement to this approach is to cluster new individuals around each previously evaluated individual and to predict the fitness values of the new individuals using the distance from the evaluated individual inside the cluster [104].

Another distance-based prediction approach is to use a rule-based system. Two IEC evaluations include individual ratings and simulated breeding or user-selection. The former has a higher searching capability but results in increased user fatigue, while the latter has the opposite characteristics. A proposed compromise is to allow a user to select better individuals and to let a rule-based system or function assign bias fitness values to unselected individuals instead of a value of 0 . One simple idea is to assign the same bias fitness values to the unselected individuals; another idea is to measure the Euclidian distances among chromosomes [57]; yet another idea is to use approximate reasoning to predict their bias fitness values from the selected individuals [135], [136]. Further improvements to the IEC-based cartoon face drawing system include measuring the distances not in a phenotype space, or face parameter space, but in a psychological space constructed by the multidimensional scaling method [192], [193], [194].

The robot control mentioned in section III-M obtains an obstacle avoidance behavior in two phases: the IEC phase where the fitness prediction model is first formed during the IEC process and the non-IEC phase that follows using the prediction model [33]. The user interacts with the individual robot by shining a light on the robot's photo sensors during the IEC phase when the robot exhibits undesired behavior. Then, the IEC constructs rules from the user preference model to determine desired robot behavior. The normal EC training phase follows the IEC phase without a real user but with a user model, shortening human interaction time and therefore reducing human fatigue.

Some research not only predicts new fitness values from the user's fitness values but also uses the predicted values for improving the display interface or screening individuals. One proposal displayed individuals roughly in the order of human evaluation and allowed IEC users to evaluate them by comparing neighboring individuals using the Euclidian distance and/or an NN to reduce human fatigue [146], [148], [149], [150], [202], [205]. When similarly evaluated individuals were grouped and displayed, it was expected to roughly and easily evaluate individuals. The application to the screening was to make an NN learn nonmusical melodies and to eliminate the non-musical melody 
from the individuals using the NN to reduce human fatigue [16].

There are many remaining research objectives for fitness prediction. One point is to construct a distance measure similar to the human evaluation scale. Euclidian distancebased fitness prediction ignores the difference in evaluation degrees among searching parameters. For example, the degree that the shapes of the eyebrows and mouth influence facial impressions is much greater than that of the hair and nose. The prediction performance depends on whether such differences are reflected into the distance measure. The second point stems from the fact that the IEC user's evaluation scale is not absolute but relative for each generation, i.e. the fitness values from generations long past and from generations recently past may be different even if the evaluation targets are the same. These differences become noise in learning the user's prediction characteristics. The simulation evaluation of the described display method in order of predicted fitness values showed a significant prediction performance, but their subjective test using human subjects has not shown the significant effectiveness in reducing human fatigue [150]. Also, it was reported that the screening of nonmusic melody by an NN, which was mentioned in previous paragraph, was very tough [16]. Further research and deep reconsideration of the prediction target or parameters are needed.

\section{Interface for Dynamic Tasks}

The fatigue problem becomes serious when an IEC user compares and evaluates sounds or movies which are spatially impossible to display as mentioned in the third IEC problem. Fatigue reduction methods using a speech processing tasks were investigated.

The first investigation concerned the number of displayed sound sources. When one sound source was used, it was easier for a user to compare the difference of several processing effects, but the user became bored and tired. The opposite effect takes place when increasing the number of sound sources. Subjective tests were conducted and compared in three cases of operability and EC convergence: (1) one sound source was used for all individuals in all generations, (2) one of 20 sources were randomly assigned to any individual, and (3) sound sources were changed at each generation, and same source was used for all individuals in same generation. The subjective tests showed that the third test was significantly better than the others [234], [235].

The second investigation was on the effect of the explicit display of an elite individual to reduce forcing the user to mentally compare the displayed sound and past sounds. Two display interfaces were evaluated by subjective tests; one indicated which was the elite individual and allowed an IEC user to play or compare the sound whenever he or she wanted, and the other was a conventional test. The subjective test results showed that this improvement of an display interface was significantly more effective [234], [235].

The third investigation was a comparison of two graphical user interfaces (GUIs): one GUI had a single play but- ton and forced the user to evaluate only the sound that the IEC GUI displayed [248], and the other test had 20 buttons that allowed an IEC user to choose any one of 20 individual sounds to be evaluated. The former test provided a simple user operation because the next sound was automatically played as soon as the user inputted a fitness rating. There was no need for the user to choose next individual to play and compare the sound because the user could not choose any individuals. The latter test's operability was good to freely compare sounds, but the number of mouse clicks for choosing an individual to play increased by one. This trade-off was evaluated through subjective tests, and it was shown that the former was more effective [235].

The GenJam in section III-C adopts real-time evaluation of melodies to shorten the total evaluation time rather than evaluation after an entire melody is played [13]. While the comparison of a displayed melody with other previous melodies took a long time and was tiresome, this real-time evaluation phrase-by-phrase was easier. This idea could be used for other IEC tasks requiring time-sequential displays such as the VR control rule acquisition of the arm wrestling robot described in section III-I.

Unlike sounds, we can evaluate simultaneously played movies and animations to certain extent if the number of simultaneous displays is only two. There is a trial to introduce this pair comparisons and a tournament selection to IEC evaluation to reduce human fatigue [110]. The advantage of the tournament selection is that the number of pair comparison is not many, which is $n-1$ for $n$ population size as same as tournament sport games, and the pair comparison is less fatigue than the absolute evaluation of one individual; its disadvantage is that the reliability of fitness values given as the winning order of the tournament is low except the winner as same as that of the tournament sport games.

\section{Acceleration of EC Convergence}

Acceleration of EC convergence significantly reduces human fatigue. Although any fast EC search methods are applicable, methods whose quick convergence in early generations are especially useful for the IEC. As previously mentioned, the practical evaluation generation of the IEC is less than first 10 or 20 generations. Quickening methods that work later, such as second order convergence of gradient methods near a global minimum, are not suitable.

A new elitist method that approximates the searching surface using a convex curve was proposed [60] and applied to the IEC [61], [149], [205]. Evaluation tests with seven benchmark functions showed the significance of a fast convergence especially in early EC generations. This idea is useful for any EC tasks whose fitness evaluation is longer than the calculation time of the convex curve (note that the geophysical simulation in section III-P takes 30 minutes to one hour in certain conditions, and fast convergence in early generation is preferable even if the quickening method takes time.) However, subjective tests have not shown that this fast convergence does not result in a 
significant reduction in human fatigue, although the only task is face drawing [149].

The human evaluation time in the IEC is extremely long from the point of view of a computer. It would be ideal to make the computer do something during this wait time to shorten the total IEC time. The idea of the pipeline-type GA is based on this idea [86], [87], [88].

\section{E. Combination of IEC and Non-IEC}

It was proposed that the parameters of fitness function are tuned by simple reinforcement learning during the interaction phase to reduce IEC user fatigue [64], [228]. It realized a system that iteratively conducted IEC and GA searches.

The damage detection system of stainless steel described in section III-K is also a combination system. It usually uses the GP and the IGP at every 100th generation [196].

\section{F. Active Intervention}

Two active user interventions into EC search were proposed: on-line knowledge embedding and Visualized IEC. The role of normal IEC users is just to evaluate the displayed sounds or images from a computer, and an optimization search itself is conducted by the EC. Research that allows the EC users to actively interfere with the EC search to accelerate the EC or IEC convergence is the topic of this section. Active intervention that motivates the users and fast EC convergence resulted by the active intervention directly reduces human fatigue.

On-line knowledge embedding is a method that provides a mechanism for accepting searching ideas, hints, or intentions during the IEC operation and allows the user to actively participate in the EC search and, hopefully, improve its convergence. For example, when a user perceives that a certain facial feature of an individual montage image will improve an EC search, the genes corresponding to the facial feature are masked to prevent crossover and mutation [25], [85], [211]. This masking means that the dimensional number of the searching space is reduced and we may be able to accelerate the EC search. Conversely, it has negative aspects that we have to pay attention, not only to entire impressions but also partial impressions, or the user's operation to specify the masked feature increases. This trade-off was evaluated using a montage system with subjective tests. The result showed that (1) the unit operation time increased $30 \%$, nevertheless the total convergence time became faster than the increased time, and (2) the obtained montage image with the on-line knowledge embedding method was significantly closer to the target face image [85], [211]. Further direct metod is to allow an IEC user to directly edit gene parameters such as SBART in section III-A.

The Visualized EC or Visualized IEC is a method that visualizes the EC search landscape by mapping the individuals with their fitness values from an $n$-D searching space to a 2-D space, helps to let the user estimate a rough global optimum position in the 2-D space, and uses the position as a new elite individual [52], [53], [215]. The Visualized EC or
IEC combines different advantages of the EC and human searching techniques; the EC directly and systematically searches the original $n$-D gene space based on EC operators, which is better than human searching techniques. Humans have an excellent capacity to grasp an entire distribution of individuals in the 2-D space at a macroscopic level that cannot be interpreted by an EC. The good news of this method is that we can expect low risk, high return; the EC convergence is dramatically improved in the best case, and only one of many individuals does not work well in the worst case.

The performances of a normal GA and the Visualized GA were compared using benchmark functions. A selforganizing map was used for the 2-D mapping. The experimental results showed that the convergence of the Visualized GA with a population size of 20 corresponds to that of normal GA with a population size from 100 to 1,000 [52], [53], [215].
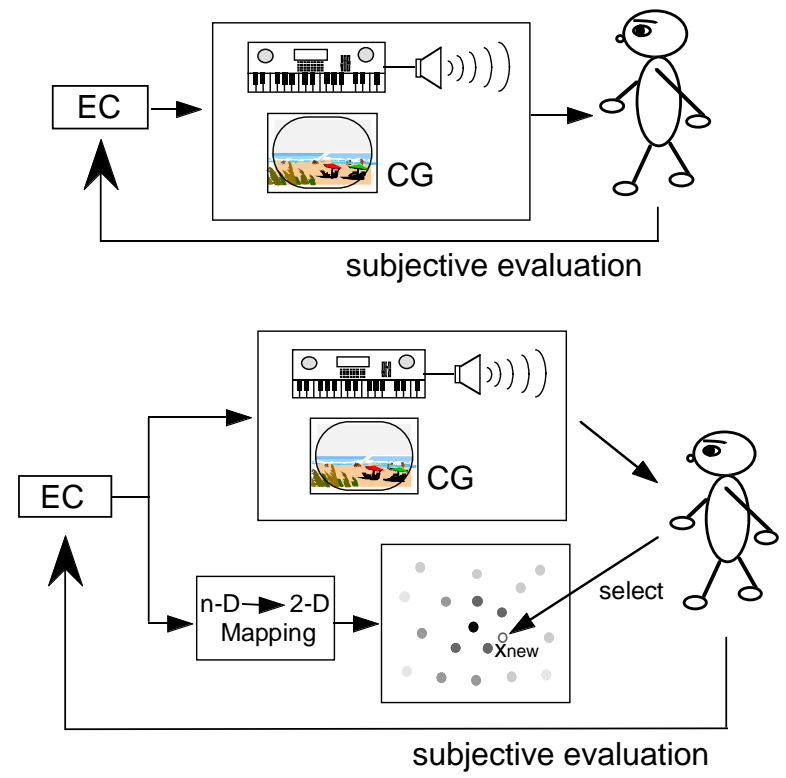

Fig. 11. Normal IEC (upper) and Visualized IEC (lower). Visualized IEC maps individuals in $n$-D EC searching space to 2 -D space and visualizes the landscape from the distribution of fitness values.

\section{G. Theoretical Research}

There was a trial to make a probabilistic model for the IEC process with stochastic Mealy automata, which was motivated by the GA modeling with the Markov chain [177]. Although this research seems to be in the early stage of the research, this IEC research is quite unique while all other IEC research has concentrated on either IEC applications or interfaces.

\section{Conclusion}

We overviewed how IEC technology has spread to a wide variety of fields, what problems remain, and what kinds of challenges need to be solved, and how to make the technology practical. 
Looking back over the history of computational intelligence, especially that of so-called Soft Computing [219]. The seeds of the NN, fuzzy systems, and EC were sown in the 1960s, and they were widely but independently researched during the 1980s; as research activities increased during the 1980s, interest in fusing them has also been rapidly increasing, and these cooperative technologies have widely and practically spread into commercial products and industrial systems during the 1990s [219]. Roughly speaking, 1980s was the decade of each computational intelligence technology, and 1990s was the decade of the cooperative technologies of computational intelligence technologies.

There are several directions where computational intelligence research could go from now. One of possible research direction would be a humanized technology where computational intelligence technologies and humans are cooperatively combined similar to the research on human factors or KANSEI in the 1990s. One of the realized ways of the humanized technology is the IEC that combines the human evaluation capability with the computer optimization capability.

The IEC will become more important in design, adjustment, and creation that are directly related with human essential KANSEI factors such as preference, emotion, feeling, and so on. It is deeply expected that as the IEC is further developed, it will help unite humanized technology with user-friendly technology.

\section{ACKNOWLEDGEMENTS}

This survey was completed with the help of several people who provided information on IEC papers, sent the papers, commented on this article, helped build a database, and prepared this paper for publication. The author is especially grateful to Professors Takao Terano and Tatsuo Unemi for their offerings of information on papers and comments on this paper and to Kei Ohnishi for his assistance to complete the author's IEC database.

\section{REFERENCES}

[1] P. J. Angeline, "Evolving fractal movies," in 1st Annual Conf. on Genetic Programming, (Stanford, CA, USA), pp. 503-511, July 1996.

[2] T. Anzai and T. Onisawa, "Acquirement of understandable fuzzy rules," in 15th Fuzzy System Symposium, (Osaka, Japan), pp. 467-470, June 1999. (in Japanese).

[3] K. Aoki, H. Takagi, and N. Fujimura, "Interactive GA-based design support system for lighting design in computer graphics," in Int. Conf. on Soft Computing (IIZUKA'96), (Iizuka, Fukuoka, Japan), pp. 533-536, World Scientific, September/October 1996.

[4] K. Aoki and H. Takagi, "3-D CG lighting design support system using interactive GA," in IEICE A-15-30, pp. 16-1-1608, Mar. 1997. (in Japanese).

[5] K. Aoki and H. Takagi, "3-D CG lighting with an interactive GA," in 1st Int. Conf. on Conventional and Knowledge-based Intelligent Electronic Systems (KES'97), (Adelaide, Australia), pp. 296-301, May 1997.

[6] K. Aoki and H. Takagi, "Lighting design support on 3-D CG," in 13th Fuzzy System Symposium, (Toyama, Japan), pp. 311314, June 1997. (in Japanese).

[7] K. Aoki and H. Takagi, "Interactive GA-based design support system for lighting design in 3-D computer graphics," Trans. of IEICE, vol. J81-DII, no. 7, pp. 1601-1608, 1998. (in Japanese).
8] E. Baker, "Summary: Evolving line drawings," in 5th Int. Conf. on Genetic Algorithm (ICGA'93), (Urbana-Champaign, IL, USA), p. 627, CA, USA: Morgan Kaufmann Publisher, 1993.

[9] E. Baker and M. Seltzer, "Evolving line drawings," in Graphics Interface '94 (W. A. Davis and B. Joe, eds.), (Banff, Alberta, Canada), pp. 91-100, CA, USA: Morgan Kaufmann Publisher, May 1994.

[10] S. Baluja, D. Pomerleau, and T. Jochem, "Simulating user's preferences: Towards automated artificial evolution for computer generated images," Tech. Rep. CMU-CS-93-198, CMU Computer Science Technical Reports, Oct. 1993.

[11] S. Baluja, D. Pomerleau, and T. Jochem, "Towards automated artificial evolution for computer-generated images," Connection Science, vol. 6, no. 2\&3, pp. 325-354, 1994.

[12] W. Banzhaf, "Interactive evolution," in Handbook of Evolutionary Computation (T. Back, D. Fogel, and Z. Michalewicz, eds.), ch. C2.10, pp. 1-5, Oxford University Press, 1997.

[13] J. A. Biles, "GenJam: A genetic algorithm for generating jazz solos," in Int. Computer Music Conf. (ICMC'94), (Aarhus, Denmark), pp. 131-137, 1994.

[14] J. A. Biles, "Al Biles Virtual Quintet, genjam." Compact disk DRK-144, 1995.

[15] J. A. Biles and W. Eign, "GenJam populi: Training an IGA via audience-mediated performance," in Int. Computer Music Conf. (ICMC'95), (Banff, Canada), pp. 347-348, 1995.

[16] J. A. Biles, P. G. Anderson, and L. W. Loggi, "Neural network fitness functions for a musical IGA," in IIA'96/SOCO'96. Int. ICSC Symposia on Intelligent Industrial Automation And Soft Computing, pp. B39-44, 1996.

[17] J. A. Biles, "Interactive GenJam: Integrating real-time performance with a genetic algorithm," in Int. Computer Music Conf. (ICMC'98), (Ann Arbor, MI, USA), pp. 232-235, Oct. 1998.

[18] J. A. Biles, "Life with GenJam: Interacting with a musical IGA," in IEEE Int. Conf. on System, Man, and Cybernetics (SMC'99), (Tokyo, Japan), pp. 652-656, Oct. 1999. vol.3.

[19] J. A. Biles, "GenJam in perspective: A tentative taxonomy for genetic algorithm music and art systems," in Genetic and Evolutionary Computation Conf. Workshop Program (GECCO2000), (Las Vegas, NV, USA), pp. 133-135, July 2000.

[20] J. A. Biles, "GenJam: Evolution of a jazz improviser," in Creative Evolutionary Systems (P. J. Bentley and D. W. Corne, eds.), Morgan Kaufman, 2000.

[21] F. Boschetti, L. Moresi, and K. Covin, "Interactive inversion in geological applications," in Int. Conf. on Knowledge-based Intelligent Information Engineering Systems (KES'99), (Adelaide, Australia), pp. 276-279, Aug./Sept. 1999.

[22] F. Boschetti and L. Moresi, "Comparison between interactive (subjective) and traditional (numerical) inversion by genetic algorithms," in Congress on Evolutionary Computation (CEC2000), (La Jolla, CA, USA), pp. 522-528, July 2000.

[23] F. Boschetti and H. Takagi, "Visualization of EC landscape to accelerate EC conversion and evaluation of its effect," in Congress on Evolutionary Computation (CEC2001), (Seoul, Korea), May 2001.

[24] F. Boschetti and L. Moresi, "Interactive inversion in geosciences," Geophysics, (in press).

[25] C. Caldwell and V. S. Johnston, "Tracking a criminal suspect through "face-space" with a genetic algorithm," in 4th Int. Conf. on Genetic Algorithm (ICGA'91), (San Diego, CA, US), pp. 416-421, CA, USA: Morgan Kaufmann Publishers, July 1991.

[26] S.-B. Cho, "Applying interactive genetic algorithm to contentbased image retrieval: A preliminary result," in Workshop on Interactive Evolutionary Computation, (Fukuoka, Japan), pp. 19-24, Mar. 1998. (in Japanese).

[27] S.-B. Cho and J.-Y. Lee, "Emotional image retrieval with interactive evolutionary computation," in Advances in Soft Computing-Engineering Design and Manufacturing (R. Roy, T. Furuhashi, and P. Chawdhry, eds.), pp. 57-66, SpringerVerlag, London, 1999.

[28] S.-B. Cho, "A human-oriented interface for emotional media manipulation with an a-life technology," Three Dimensional Images, vol. 13, no. 3, pp. 71-76, 1999.

[29] S. Das, T. Franguiadakis, M. Papka, T. A. DeFanti, et al., "A genetic programming application in virtual reality," in 1st IEEE Conf. on Evolutionary Computation (ICEC'94), vol. 1, (Orlando, FL, USA), pp. 480-484, IEEE, June 1994. 
[30] R. Dawkins, The Blind Watchmaker. Essex: Longman, 1986.

[31] R. Dawkins, "The evolution of evolvability," in Artificial Life (C. G. Langton, ed.), pp. 201-220, Addison-Wesley, 1989.

[32] T. Disz, M. E. Papka, and R. Stevens, "UbiWorld: An environment integrating virtual reality, supercomputing and design," in 6th Heterogeneous Computing Workshop (HCW'97) (D. Hensgen, ed.), (Geneva, Switzerland), pp. 46-57, IEEE Computer Society Press, Apr. 1997.

[33] G. Dozier, "Evolving robot behavior via interactive evolutionary computation: From real-world to simulation," in 16th ACM Symposium on Applied Computing (SAC2001), (Las Vegas, USA), pp. 340-344, Mar. 2001.

[34] I. K. C. Eckert and M. Stacey, "Interactive generative systems for conceptual design: an empirical perspective," (AI EDAM) Artificial Intelligence for Engineering Design, Analysis and Manufacturing, vol. 13, no. 4, pp. 303-320, 1999.

[35] H. Esbensen, "EXPLORER: An interactive floorplanner for design space exploration," in European Design Automation Conf. (EURO-DAC'96) with EURO-VHDL'96 and Exhibition, (Geneva, Switzerland), pp. 356-361, Sept. 1996.

[36] B. Filipic and D. Juricic, "An interactive genetic algorithm for controller parameter optimization," in Int. Conf. on Artificial Neural Nets and Genetic Algorithms, (Innsbruck, Austria), pp. 458-62, Apr. 1993.

[37] M. Fujii and T. Furuhashi, "A study of interactive between humans and systems using fuzzy classifier system," in Workshop on Interactive Evolutionary Computation, (Fukuoka, Japan), pp. 37-41, Mar. 1998. (in Japanese).

[38] S. Fujii, H. Takagi, M. Ohsaki, and Y. Arai, "Knowledge acquisition for compensation for hearing impairment based on IEC Fitting," in Technical Report of ASJ, H-99-121, (Kumamoto, Japan), Dec. 1999. (in Japanese).

[39] S. Fujii, H. Takagi, M. Ohsaki, M. Watanabe, and S. Sakamoto, "Evaluation and analysis of IEC Fitting," in 7th Western Pacific Regional Acoustics Conf. (WESTPRAC VII), (Kumamoto, Japan), pp. 369-372, Oct. 2000.

[40] H. Furuta, K. Maeda, and E. Watanabe, "Application of genetic algorithm to aesthetic design of bridge structures," $M i$ crocomputers in Civil Engineering, vol. 10, no. 6, pp. 415-421, 1995.

[41] R. Gatarski, "Evolutionary banners exploring a generative design approach," in Generative Art (GA'98), (Milan, Italy), pp. 221-240, Dec. 1998.

[42] R. Gatarski, "Evolutionary banners: An experiment with automated advertising design," in Conference on Telecommunications and Information Markets (COTIM'99), (Providence, R.I., USA.), Sept. 1999.

[43] D. E. Goldberg, W. B. Hall, L. Krussow, E. Lee, and A. Walker, "Teamwork for a quality education: Low-cost, effective educational reform, through department-wide competition of teams," IlliGAL Report 98005, University of Illinois, UrbanaChampaign, Feb. 1998.

[44] J. Graf and W. Banzhaf, "Interactive evolution of images," in 4th Annual Conf. on Evolutionary Programming (J. R. McDonnell, R. G. Reynolds, and D. B. Fogel, eds.), (San Diego, CA, USA), pp. 53-65, MIT Press, Mar. 1995.

[45] J. Graf, "Interactive evolutionary algorithms in design," in Int. Conf. on Artificial Neural Nets and Genetic Algorithms, (Alès, France), pp. 227-230, Apr. 1995.

[46] J. Graf and W. Banzhaf, "Interactive evolution for simulated natural evolution," in Artificial Evolution. European Conf. (AE'95). Selected Papers. (J. M. Alliot, E. Lutton, E. Ronald, M. Schoenauer, et al., eds.), pp. 259-272, Berlin, Germany: Springer-Verlag, Sept. 1995.

[47] J. Graf and W. Banzhaf, "An expansion operator for interactive evolution," in IEEE Int. Conf. on Evolutionary Computation (ICEC'95), vol. 2, (Perth, WA, Australia), pp. 798-802, IEEE, Nov./Dec. 1995.

[48] J. Graf, "Interactive evolution: A prototyping framework for engineering," Microcomputers in Civil Engineering, vol. 11, no. 3, pp. 185-193, 1996.

[49] G. R. Greenfield, "New directions for evolving expressions," in 1st Annual Conf. of BRIDGES: Mathematical Connections in Art, Music, and Science, (Winfield, Kansas, USA), July 1998.

[50] G. R. Greenfield, "Evolving expressions and art by choice," Leonard, vol. 33, no. 2, pp. 93-99, 2000.

[51] F. Gruau and K. Quatramarau, "Cellular encoding for interac- tive evolutionary robotics," Cognitive Science Research Paper 425, University of Sussex, July 1996.

[52] N. Hayashida and H. Takagi, "Introduction of an active selection to the interactive evolutionary computation by using multidimensional data visualization," in SOFT Kyushu Chapter Meeting, (Saga, Japan), pp. 17-20, Dec. 1999. (in Japanese).

[53] N. Hayashida and H. Takagi, "Visualized IEC: Interactive evolutionary computation with multidimensional data visualization," in Industrial Electronics, Control and Instrumentation (IECON2000), (Nagoya, Japan), pp. 2738-2743, Oct. 2000.

[54] M. Herdy, "Evolution strategies with subjective selection," in Int. Conf. on Evolutionary Computation (ICEC/PPSN IV), (Berlin, Germany), pp. 22-26, 1996.

[55] M. Herdy, "Evolutionary optimization based on subjective selection - evolving blends of coffee," in 5th European Congress on Intelligent Techniques and Soft Computing EUFIT'97, (Aachen), pp. 640-644, Sept. 1997.

[56] D. Horowitz, "Generating rhythms with genetic algorithms," in Int. Computer Music Conf. (ICMC'94), (Aarhus, Denmark), pp. 142-143, 1994.

[57] F.-C. Hsu and J.-S. Chen, "A study on multi criteria decisionmaking model: Interactive genetic algorithms approach," in IEEE Int. Conf. on System, Man, and Cybernetics (SMC'99), (Tokyo, Japan), pp. 634-639, Oct. 1999. vol.3.

[58] T. Igarashi, S. Matsuoka, and T. Masui, "Adaptive recognition of implicit structures in human-organized layouts," in 11th IEEE Int. Symposium on Visual Languages (V. Haarslev, ed.), (Darmstadt, Germany), pp. 258-66, IEEE Comput. Soc. Press, Sept. 1995

[59] M. Inada and T. Terano, "Analyzing clinical data using interactive evolutionary computation and inductive learning," in 26th Intelligent System Symposium of SICE, (Yokohama, Japan), Mar. 1999. (in Japanese).

[60] T. Ingu, H. Takagi, and M. Ohsaki, "Improvement of interface for interactive genetic algorithms -proposal for fast GA convergence-," in 13th Fuzzy System Symposium, (Toyama, Japan), pp. 859-862, June 1997. (in Japanese).

[61] T. Ingu and H. Takagi, "Accelerating a GA convergence by fitting a single-peak function," in IEEE Int. Conf. on Fuzzy Systems (FUZZ-IEEE'99), (Seoul, Korea), pp. 1415-1420, Aug. 1999.

[62] T. Inoue, T. Furuhashi, M. Fujii, et al., "Development of nurse scheduling support system using interactive EA," in IEEE Int. Conf. on Systems, Man, and Cybernetics (SMC'99), (Tokyo, Japan), pp. 533-537, October 1999. vol.5.

[63] Y. Ishino, K. Yoshinaga, and T. Terano, "Data analyses using simulated breeding and inductive learning techniques," in 21st Intelligent System Symposium, (Tokyo, Japan), pp. 1-6, May 1995. (in Japanese).

[64] Y. Ishino and T. Terano, "Marketing data analysis using simulated breeding and inductive learning techniques," J. of Japan Society for Artificial Intelligence, vol. 12, no. 1, pp. 121-131, 1997. (in Japanese).

[65] Y. Ishino, K. Hori, and S. Nakasuka, "Interactive knowledge acquisition for concept development of consumer products," in 3rd Int. Conf. on Knowledge-Based Intelligent Information Engineering Systems (KES'99), (Adelaide, SA, Australia), pp. 272-275, Aug./Sept. 1999.

[66] Y. Ishino, K. Hori, and S. Nakasuka, "Strategic concept formation of consumer goods based on knowledge acquisition from questionnaire data," in IEEE Int. Conf. on Systems, Man, and Cybernetics (SMC'99), (Tokyo, Japan), pp. 533-537, Oct. 1999. vol.5.

[67] K. Isono, M. Oda, and S. Akamatsu, "A facial image generating system based on a genetic algorithm-gene rating subjective impression," Transactions of the Institute of Electronics, Information and Communication Engineers D-II, vol. J82D-II, no. 3, pp. 483-493, 1999. (in Japanese).

[68] E. Ito and S. Ishizaki, "Creative design support system using evolutionary computation," in 2nd Int. Conf. on Cognitive Science (ICCS'99), (Tokyo, Japan), pp. 657-660, 1999.

[69] M. Iwai, "Adjustment of FM operator tones by genetic algorithm," in Summer Program Symposium on Amusement and Computer, July 1994. (in Japanese).

[70] T. Iwasaki, A. Kimura, Y. Todoroki, Y. Hirose, H. Takagi, and T. Takeda, "Interactive virtual aquarium (1st report)," in 5th Annual Conf. of the Virtual Reality Society of Japan, pp. 141144, Sept. 2000. (in Japanese). 
[71] I. Kamihira, M. Yamaguchi, and H. Kita, "Online adaptation of vehicles by means of an evolutionary control system," in IEEE Int. Conf. on Systems, Man, and Cybernetics (SMC'99), (Tokyo, Japan), pp. 553-558, Oct. 1999.

[72] S. Kamohara, H. Takagi, and T. Takeda, "Control rule acquisition for arm wrestling in virtual reality," in 12th Fuzzy System Symposium, (Tokyo, Japan), pp. 487-490, June 1996. (in Japanese).

[73] S. Kamohara, H. Takagi, and T. Takeda, "Control rule acquisition for an arm wrestling robot," in IEEE Int. Conf. on System, Man, Cybernetics (SMC'97), vol. 5, (Orlando, FL, USA), pp. 4227-4231, Oct. 1997.

[74] S. Kato, "Feature selection in flexible retrieval," Transactions of the Institute of Electronics, Information and Communication Engineers D-II, vol. J80D-II, no. 2, pp. 598-606, 1997. (in Japanese).

[75] S. Kato and S. Iisaku, "An image retrieval method based on a genetic algorithm," in Twelfth Int. Conf. on Information Networking (ICOIN-12), (Tokyo, Japan), pp. 333-336, Jan. 1998.

[76] N. Katsuyama and H. Yamakawa, "A study on heredity and evolution of designs considering KANSEI by using genetic algorithms," in Japan Society of Mechanical Engineering 3rd Annual Meeting of Design Engineering 85 System, (Tokyo, Japan), pp. 43-47, May 1993. (in Japanese).

[77] Y. Katurada and Y. Maeda, "Support system for automatically emotional generation using interactive EC," in 16th Fuzzy System Symposium, (Akita, Japan), pp. 297-300, Sept. 2000. (in Japanese).

[78] H.-S. Kim and S.-B. Cho, "Development of an IGA-based fashion design aid system with domain specific knowledge," in Proc. of IEEE SMC'99, (Tokyo, Japan), pp. III-663-III-668, Oct. 1999.

[79] H.-S. Kim and S.-B. Cho, "Genetic algorithm with knowledgebased encoding for interactive fashion design," in Lecture Notes in Artificial Intelligence, Springer Valg, 2000. no. 1886, pp. 404-414.

[80] H.-S. Kim and S.-B. Cho, "An emotion-based fashion design aid system using interactivity," the Korea Information Science Society (B), vol. 27, no. 9, pp. 942-951, 2000. (in Korean).

[81] H.-S. Kim and S.-B. Cho, "Knowledge-based encoding in interactive genetic algorithm for a fashion design aid system," in Proc. of GECCO, Poster Papers, p. 757, July 2000.

[82] H.-S. Kim and S.-B. Cho, "Application of interactive genetic algorithm to fashion design," Engineering Applications of Artificial Intelligence, vol. 13, no. 6, pp. 635-644, 2000.

[83] A. Kimura, T. Iwasaki, Y. Kitajima, H. Takagi, and T. Takeda, "Development of fish shape generator based on interactive evolutionary computation," in Joint Technical Meeting of Kyushu Chapters of Electric Related Societies, (Fukuoka, Japan), p. 525, September 2000. (in Japanese).

[84] K. Kishi and H. Takagi, "Developing montage system for study on interactive evolutionary computation," in SOFT 3rd Workshop on Evaluation of Heart and Mind, (Gamagoori, Aichi, Japan), pp. 15-18, Nov. 1998. (in Japanese).

[85] K. Kishi and H. Takagi, "Evaluation of on-line knowledge embedding for interactive evolutionary computation," in 15th Fuzzy System Symposium, (Osaka, Japan), pp. 379-380, June 1999. (in Japanese).

[86] A. Kitamoto and M. Takagi, "Learning criteria for similaritybased image retrieval using simulated breeding by pipeline-type genetic algorithms," in Technical Report of IEICE, HIP96-4, pp. 17-22, June 1996. (in Japanese).

[87] A. Kitamoto and M. Takagi, "Interactive image browsing using pipeline-type genetic algorithm," in Workshop on Interactive Evolutionary Computation, (Fukuoka, Japan), pp. 31-36, Mar. 1998. (in Japanese).

[88] A. Kitamoto and M. Takagi, "Interactive image browsing using queue-based genetic algorithm," J. of Japanese Society for Artificial Intelligence, vol. 13, no. 5, pp. 728-738, 1998. (in Japanese).

[89] M. Korenaga and M. Hagiwara, "An interior layout support system with interactive evolutionary computation," Transactions of the Information Processing Society of Japan, vol. 41, no. 11, pp. 3152-3160, 2000. (in Japanese).

[90] A. Kosorukoff and D. Goldberg, "Genetic algorithms for social innovation and creativity," IlliGAL Report 2001005, University of Illinois, Urbana-Champaign, Jan. 2001. system with tree structure representation," in 16th Fuzzy System Symposium, (Akita, Japan), pp. 327-330, Sept. 2000. (in Japanese).

[92] J. Kotani and M. Hagiwara, "An evolutionary design-supportsystem with structural representation," in IEEE Int. Conf. on Industrial Electronics, Control and Instrumentation 2000 (IECON2000), (Nagoya, Japan), pp. 672-677, Oct. 2000.

[93] N. Kubota, K. Watanabe, and F. Kojima, "Trajectory generation of human-friendly robots using interactive genetic algorithm," in 15th Fuzzy System Symposium, (Osaka, Japan), pp. 355-358, June 1999. (in Japanese).

[94] N. Kubota, K. Watanabe, and F. Kojima, "Interactive genetic algorithm for trajectory generation of human friendly robots," Trans. of the Japan Society of Mechanical Engineers, Series $C$, vol. 66, no. 647, pp. 2274-2279, 2000. (in Japanese).

[95] N. Kubota, Y. Nojima, N. Baba, F. Kojima, and T. Fukuda, "Evolving pet robot with emotional model," in Congress on Evolutionary Computation (CECO0), (La Jolla, CA, USA), pp. 1231-1237, July 2000.

[96] K. Kuriyama and T. Terano, "Interactive story composition support by genetic algorithms," in World Conf. on Artificial Intelligence in Education. (AI-ED'97), (Kobe, Japan), pp. 615-617, Aug. 1997.

[97] K. Kuriyama, T. Terano, and M. Numao, "Authoring support by interactive genetic algorithm and case based reasoning," in Workshop on Interactive Evolutionary Computation, (Fukuoka, Japan), pp. 13-18, Mar. 1998. (in Japanese).

[98] K. Kuriyama, T. Terano, and M. Numao, "Authoring support by interactive genetic algorithm and case base retrieval," in IEEE 2nd Int. Conf. on Conventional and Knowledge-based Intelligent Electronic Systems (KES'98), (Adelaide, SA, Australia), pp. pp.485-488, Apr. 1998.

[99] K. Kuriyama, T. Terano, and M. Numao, "Authoring support by interactive genetic algorithm and case base retrieval," in Asia Fuzzy System Symposium, (Masan, Korea), pp. pp.485488, June 1998.

[100] J.-Y. Lee and S.-B. Cho, "Interactive genetic algorithm for content-based image retrieval," in Asia Fuzzy System Symposium (AFSS'98), (Masan, Korea), pp. pp.479-484, June 1998.

[101] J.-Y. Lee and S.-B. Cho, "Interactive genetic algorithm with wavelet coefficients for emotional image retrieval," in Proc. Int. Conf. on Soft Computing, (Iizuka, Fukuoka, Japan), pp. 829832, Oct. 1998.

[102] J.-Y. Lee and S.-B. Cho, "Application of interactive genetic algorithm to image retrieval based on emotion," the Korea Information Science Society (B), vol. 26, no. 3, pp. 422-430, 1999. (in Korean).

[103] J.-Y. Lee and S.-B. Cho, "Incorporating human preference into content-based image retrieval using interactive genetic algorithm," in Proc. of GECCO'99, Poster Papers, p. 1788, July 1999.

[104] J.-Y. Lee and S.-B. Cho, "Sparse fitness evaluation for reducing user burden in interactive genetic algorithm," in Proc. of FUZZ-IEEE'99, pp. II-998-II1003, Aug. 1999.

[105] D. Levine, M. Facello, P. Hallstrom, et al., "Stalk: an interactive system for virtual molecular docking," IEEE Computational Science and Engineering, vol. 4, no. 2, pp. 55-65, 1997.

[106] M. A. Lewis, A. H. Fagg, and A. Solidum, "Genetic programming approach to the construction of a neural network for control of a walking robot," in IEEE Int. Conf. on Robotics and Automation, vol. 3, (Nice, France), pp. 2618-2623, May 1992.

[107] I. S. Lim, "Evolving facial expressions," in IEEE Int. Conf. on Evolutionary Computation (ICEC'95), (Perth, WA, Australia), pp. 515-520, Nov./Dec. 1995. vol.2.

[108] I. S. Lim, "Evolutionary art in virtual world," in 2nd EUROGRAPHICS Workshop on Virtual Environments, (Monte Carlo, Monaco), 1995.

[109] I. S. Lim and D. Thalmann, "Pro-actively interactive evolution for computer animation," in Eurographics Workshop on Animation and Simulation (CAS'99), (Milan, Italy), pp. 45-52, Sept. 1999.

[110] I. S. Lim and D. Thalmann, "Tournament selection for browsing temporal signals," in ACM Symposium on Applied Computing (SAC2000), (Como, Italy), pp. 570-573, Mar. 2000.

[111] S. J. Louis and R. Tang, "Interactive genetic algorithms for the traveling salesman problem," in Genetic and Evolution- 
ary Computation Conf. (ICGA-99), (Orlando, FL, USA), pp. 1043-1048, July 1999. vol.1.

[112] H. H. Lund, L. Pagliarini, and P. Miglino, "Artistic design with GA \& NN," in 1st Nordic Workshop on Genetic Algorithms and Their Applications (1NWGA), (Vaasa, Finland), pp. 97105, Jan. 1995.

[113] H. H. Lund and O. Miglino, "Evolving and breeding robots," in 1st European Workshop on Evolutionary Robotics (EboRobot98), (Berlin, Germany), pp. 192-210, SpringerVerlag, Apr. 1998.

[114] H. H. Lund, O. Miglino, L. Pagliarini, A. Billard, and A. Ijspeert, "Evolutionary robotics - a children's game," in IEEE Int. Conf. on Evolutionary Computation (ICEC'98), pp. 154-158, May 1998.

[115] J. Martinez and S. Marchand, "Towards intelligent retrieval in image databases," in Int. Workshop on Multi-Media Database Management Systems, (Dayton, OH, USA), pp. 38-45, Aug. 1998.

[116] T. Masui, "Interactive graphic object layout with genetic algorithms," in Human Interface, 41-6, pp. 41-48, Mar. 1992. (in Japanese).

[117] T. Masui, "Graphic object layout with interactive genetic algorithms," in IEEE Workshop on Visual Languages, (Los Alamitos, CA, USA), pp. 74-80, 1992.

[118] T. Masui, "Evolutionary learning of graph layout constraints from examples," in UIST'94. 7th Annual Symposium on User Interface Software and Technology, (Marina del Rey, CA, USA), pp. 103-8, ACM, Nov. 1994.

[119] J. McCormack, "Interactive evolution of L-system grammars for computer graphics modelling," in Complex Systems: from Biology to Computation (D. G. Green and T. Bossomaier, eds.), pp. 118-130, Amsterdam, Netherlands: IOS Press, Aug./Sept. 1993.

[120] T. Miyazaki and M. Hagiwara, "A poster creating support system to reflect Kansei," Transactions of the Information Processing Society of Japan, vol. 38, no. 10, pp. 1928-1936, 1997. (in Japanese).

[121] N. Monmarché, G. Nocent, M. Slimane, G. Venturini, and P. Santini, "Imagine: a tool for generating HTML style sheets with an interactive genetic algorithm based on genes frequencies," in IEEE Int. Conf. on System, Man, and Cybernetics (SMC'99), (Tokyo, Japan), pp. 640-645, Oct. 1999. vol.3.

[122] T. Morita, H. Iba, and M. Ishizuka, "Generating emotional voice and behavior expression by interactive evolutionary computation," in 62nd Annual Meeting of Japan Society for Information Processing, (Yokohama, Japan), pp. 45-46, Mar. 2001. (in Japanese).

[123] A. Moroni, J. Manzolli, F. von Zuben, and R. Gudwin, "Evolutionary computation applied to algorithmic composition," in Congress on Evolutionary Computation (CEC'99), (Washington, DC, USA), pp. 807-811, July 1999. vol.2.

[124] T. Mutoh, N. Komagata, and K. Ueda, "An experimental study for automatically generating image filter sequence by using simulated breeding," in Workshop on Interactive Evolutionary Computation, (Fukuoka, Japan), pp. 7-12, Mar. 1998. (in Japanese).

[125] S. Nabeta and T. Terano, "Design support system about timevarying multimedia titles by interactive genetic algorithm," in 12th Annual Conf. of JSAI, (Yokohama, Japan), pp. 489-490, June 1998. (in Japanese).

[126] M. Nagao, M. Yamada, K. Suzuki, and A. Ohuchi, "Evaluation of the image retrieval system using interactive evolutionary computation," in Workshop on Interactive Evolutionary Computation, (Fukuoka, Japan), pp. 25-30, Mar. 1998. (in Japanese).

[127] M. Nagao, M. Yamamoto, K. Suzuki, and A. Ohuchi, "Evaluation of the image retrieval system using interactive genetic algorithm," J. of Japanese Society for Artificial Intelligence, vol. 13, no. 5, pp. 720-727, 1998. (in Japanese).

[128] Y. Nakanishi, "Applying evolutionary systems to design aid system," in ALIFE V, Poster Presentations, pp. PP-25, 147154, May 1996.

[129] Y. Nakanishi, "Capturing preference into a function using interactions with a manual evolutionary design aid system," in Late Breaking Papers at Genetic Programming, pp. 133-140, July 1996.

[130] Y. Nakanishi, "Control and evaluation of interactive evolutionary system with a preference function: Applying genetic pro- gramming to design aid system," J. of Japanese Society for Artificial Intelligence, vol. 13, no. 5, pp. 704-711, 1998. (in Japanese).

[131] G. L. Nelson, "Sonomorphs: An application of genetic algorithms to growth and development of musical organisms," in 4th Biennial Art 83 Technology Symposium, (New London, CT, USA), pp. 155-169, Mar. 1993.

[132] G. L. Nelson, "Further adventures of the sonomorphs," in 5th Biennial Art \&5 Technology Symposium, (New London, CT, USA), pp. 51-64, Mar. 1995.

[133] T. C. Nguyen and T. S. Huang, "Evolvable modeling: Design adaptation through hierarchical evolution for 3-D model-based vision." TCGA File No.02852, (unpublished tech. report), 1993.

[134] T. C. Nguyen and T. S. Huang, "Evolvable 3D modeling for model-based object recognition systems," in Advances in GP, ch. 22, pp. 459-475, Cambridge, MA, USA: MIT Press, 1994.

[135] K. Nishio, M. Murakami, E. Mizutani, and N. Honda, "Efficient fuzzy fitness assignment strategies in an interactive genetic algorithm for cartoon face search," in 6th Int. Fuzzy Systems Association World Congress (IFSA'95), vol. 1, (Sao Pãulo), pp. 173-176, July 1995.

[136] K. Nishio, M. Murakami, E. Mizutani, and N. Honda, "Fuzzy fitness assignment in an interface genetic algorithm for a cartoon face search," in Genetic Algorithm and Fuzzy Logic Systems, Soft Computing Perspectives (E. Sanchez, T. Shibata, and L. A. Zadeh, eds.), vol. 7 of Advances in Fuzzy Systems Applications and Theory, pp. 175-192, World Scientific, 1997.

[137] H. Nishino, H. Takagi, and K. Utsumiya, "A digital prototyping system for designing novel 3D geometries," in 6th Int. Conf. on Virtual Systems and MultiMedia (VSMM2000), (Ogaki, Gifu, Japan), pp. 473-482, Oct. 2000.

[138] H. Nishino, H. Takagi, and K. Utsumiya, "3D modeling support mechanism by using interactive evolutionary computation," in Tech. Report of IEICE, Vol.100, No.461, IE2000-78, pp. 1-8, Nov. 2000. (in Japanese).

[139] H. Nishino, H. Takagi, S.-B. Cho, and K. Utsumiya, "A 3D modeling system for creative design," in The 15th Int. Conf. on Information Networking (ICOIN-15), (Beppu, Japan), pp. 479-486, Jan./Feb. 2001.

[140] T. Noda, H. Takagi, and M. Zhang, "Construction of a psychological factor space for an IGA-based image retrieval system and subjective test for the system," in 15th Fuzzy System Symposium, (Osaka, Japan), pp. 683-684, June 1999. (in Japanese).

[141] T. Noda, D. Zhao, H. Takagi, and A. Teraoka, "Media database retrieval and conversion system based on impression," in SOFT Kyushu Chapter Meeting, (Saga, Japan), pp. 1-4, Dec. 1999. (in Japanese).

[142] T. Noda, D. Zhao, and H. Takagi, "Music database retrieval and media conversion system based on impression," in 6th Int. Conf. on Soft Computing (IIZUKA2000), (Iizuka, Fukuoka, Japan), pp. 151-156, Oct. 2000.

[143] U.-M. O'Reilly and G. Ramachandran, "A preliminary investigation of evolution as a form design strategy," in Artificial Life VI (C. Adami, R. Belew, H. Kitano, and C. Taylor, eds.), (Los Angeles, CA, UAS), pp. 443-447, MIT Press, June 1998.

[144] T. Obata and M. Hagiwara, "A color poster creating support system to reflect Kansei," Transactions of the Information Processing Society of Japan, vol. 41, no. 3, pp. 701-710, 2000. (in Japanese).

[145] G. Ochoa, "On genetic algorithms and Lindenmayer systems," in 5th Int. Conf. on Parallel Problem Solving from Nature (PPSN V), (Amsterdam, Netherlands), pp. 335-344, Sept. 1998.

[146] M. Ohsaki and H. Takagi, "Application of interactive evolutionary computation to digital hearing aids fitting," in 14th Fuzzy System Symposium, (Gifu, Japan), pp. 193-194, June 1998. (in Japanese).

[147] M. Ohsaki, H. Takagi, and K. Ohya, "An input method using discrete fitness values for interactive GA," J. of Intelligent and Fuzzy Systems, vol. 6, pp. 131-145, 1998.

[148] M. Ohsaki and H. Takagi, "Reduction of the fatigue of human interactive EC operators-improvement of present interface by prediction of evaluation order," J. of Japanese Society for Artificial Intelligence, vol. 13 , no. 5, pp. $712-719$, 1998. (in Japanese).

[149] M. Ohsaki, H. Takagi, and T. Ingu, "Methods to reduce the human burden of interactive evolutionary computation," in 
Asia Fuzzy System Symposium (AFSS'98), (Masan, Korea), pp. 495-500, June 1998

[150] M. Ohsaki and H. Takagi, "Improvement of presenting interface by predicting the evaluation order to reduce the burden of human interactive EC operators," in IEEE Int. Conf. on System, Man, Cybernetics (SMC'98), (San Diego, CA, USA), pp. 1284-1289, Oct. 1998.

[151] M. Ohsaki and H. Takagi, "Application of interactive evolutionary computation to optimal tuning of digital hearing aids," in Int. Conf. on Soft Computing (IIZUKA'98), (Iizuka, Fukuoka, Japan), pp. 849-852, World Scientific, Oct. 1998.

[152] M. Ohsaki, T. Tsumura, H. Takagi, and M. Shimada, "Evaluation of IEC fitting system on speech hearing," in Annual Meeting of Acoustic Society of Japan, 1-2-19, pp. 361-362, Mar. 1999. (in Japanese).

[153] M. Ohsaki, T. Tsumura, H. Takagi, and M. Shimada, "Application of IEC fitting system to music hearing," in Annual Meeting of Acoustic Society of Japan, 1-2-20, pp. 363-364, Mar. 1999. (in Japanese).

[154] M. Ohsaki and H. Takagi, "Evaluation of an interactive ECbased fitting system for digital hearing aids," in 15th Fuzzy System Symposium, (Osaka, Japan), pp. 381-384, June 1999. (in Japanese).

[155] M. Ohsaki, H. Takagi, M. Watanabe, and S. Sakamoto, "Development and evaluation of an IEC-based hearing aids fitting system for practical use - application to a conventional hearing aid," in Annual Meeting of Acoustic Society of Japan, 2-10-1, pp. 331-332, Mar. 2000. (in Japanese).

[156] M. Ohsaki and H. Takagi, "Design and development of an IECbased hearing aids fitting system," in 4th Asia Fuzzy System Symposium (AFSS'00), (Tsukuba, Japan), pp. 543-548, June 2000 .

[157] M. Ohsaki and H. Takagi, "Human interface of interactive evolutionary computation and its evaluation," in Genetic Algorithms 4 (H. Kitano, ed.), ch. 13, pp. 397-438, Sangyo Publisher, Aug. 30, 2000. (in Japanese).

[158] T. Onisawa and T. Anzai, "Acquisition of intelligible fuzzy rules," in IEEE Int. Conf. on Systems, Man, and Cybernetics (SMC'99), (Tokyo, Japan), pp. 268-273, Oct. 1999. vol.5.

[159] T. Onisawa and T. Anzai, "Acquisition of intelligible fuzzy rules with interaction," in 16th Fuzzy System Symposium, (Akita, Japan), pp. 451-454, Sept. 2000. (in Japanese).

[160] T. Onisawa, W. Takizawa, and M. Unehara, "Composition of melody reflecting user's feeling," in IEEE Int. Conf. on Industrial Electronics, Control and Instrumentation (IECON2000), (Nagoya, Japan), pp. 2738-2743, Oct. 2000.

[161] K. Otoba, K. Tanaka, and M. Hitafuji, "Image processing and interactive selection with Java based on genetic algorithms," in 3rd IFAC/CIGR Workshop on Artificial Intelligence in Agriculture, (Makuhari, Japan), pp. 83-88, Apr. 1998.

[162] L. Pagliarini, H. H. Lund, O. Miglino, and D. Parisi, "Artificial Life: A new way to build educational and therapeutic games," in ALIFE $V$, pp. 152-156, MIT Press, 1996.

[163] L. Pagliarini, A. Dolan, F. Menczer, and H. H. Lund, "ALife meets web: Lessons learned," in 1st Int. Conf. on Virtual World (VW'98), (Berlin, Germany), pp. 156-167, SpringerVerlag, July 1998.

[164] M. E. Papka and R. Stevens, "Ubiworld: An environment integrating virtual reality, supercomputing and design," in 5th IEEE Int. Symposium on High Performance Distributed Computing, (Syracuse, NY, USA), pp. 306-307, IEEE Comput. Soc. Press, Aug. 1996.

[165] I. C. Parmee, "The concrete arch dam: An evolutionary model of the design process," in Int. Conf. on Artificial Neural Nets and Genetic Algorithms, (Innsbruck, Austria), pp. 544-551, Apr. 1993.

[166] I. C. Parmee and C. R. Bonham, "The maintenance of search diversity for effective design space decomposition using cluster oriented genetic algorithms (COGAs) and multi-agent strategies (GAANT)," in Adaptive Computing in Engineering Design and Control (I. C. Parmee, ed.), (Plymouth, UK), pp. 128-138, 1996.

[167] I. C. Parmee, "Strategies for the integration of evolutionary algorithms (COGAs) for the identification of high-performance regions design spaces," in Evolutionary Algorithms in Engineering Applications (D. Dasgupta and Z. Michelewicz, eds.), pp. 453-478, Berlin, Germany: Springer-Varlag, 1996.

[168] I. C. Parmee and C. R. Bonham, "Supporting innovative and creative design using interactive designer/evolutionary computing strategies," in Computation Models of Creative Design Conf. (J. Gero and M. Maher, eds.), (Sydney, Australia), 1998.

[169] I. C. Parmee and C. R. Bonham, "Cluster-oriented genetic algorithms to support interactive designer/evolutionary computing systems," in Congress on Evolutionary Computation (CEC'99), vol. 1, (Washington, DC, USA), pp. 546-553, July 1999.

[170] I. C. Parmee, "Exploring the design potential of evolutionary search, exploration and optimization," in Evolutionary Design by Computer, (San Francisco, CA,USA), Morgan Kaufmann, 1999.

[171] I. C. Parmee and C. R. Bonham, "Towards the support of innovative conceptual design through interactive designer/evolutionary completing strategies," (AI EDAM) Artificial Intelligence for Engineering Design, Analysis and Manufacturing, vol. 14, no. 1, pp. 3-16, 2000.

[172] I. C. Parmee, A. H. Watson, D. Cvetković, and C. R. Bonham, "Multiobjective satisfaction within an interactive evolutionary design environment," Evolutionary Computation, vol. 8, no. 2, pp. 197-222, 2000.

[173] I. C. Parmee, "Developing the framework for an interactive evolutionary design system," in 5th Joint Conf. on Information Sciences (JCIS2000), (Atlantic City, NJ, USA), pp. 991-994, Feb./Mar. 2000.

[174] R. Poli and S. Cagnoni, "Genetic programming with userdriven selection: Experiments on the evolution of algorithms for image enhancement," in 2nd Annual Conf. on Genetic Programming, pp. 269-277, 1997.

[175] F. Raynal, E. Lutton, P. Collet, and M. Schoenauer, "Manipulation of non-linear IFS attractors using genetic programming," in Congress on Evolutionary Computation (CEC'99), (Washington, DC, USA), pp. 1171-1177, July 1999. vol.2.

[176] A. Rowbottom, "Evolutionary art and form," in Evolutionary Design by Computers (P. Bentley, ed.), ch. 11, pp. 260-277, CA, USA: Morgan Kaufmann Publishers, 1999.

[177] G. Rudolph, "On interactive evolutionary algorithms and stochastic Mealy automata," in 4th Parallel Problem Solving from Nature - PPSN IV. Int. Conf. on Evolutionary Computation, (Berlin, Germany), pp. 218-226, Sept. 1996.

[178] T. Sato and M. Hagiwara, "Tool creating support system using evolutionary techniques," in 15th Fuzzy System Symposium, (Osaka, Japan), pp. 363-366, June 1999. (in Japanese).

[179] T. Sato and M. Hagiwara, "IDSET: Interactive design system using evolutionary techniques," J. of Computer-Aided Design, (accepted).

[180] Y. Sato, "Voice conversation using evolutionary computation of prosodic control," in 12th Symposium on Human Interface, (Yokohama, Japan), pp. 469-475, Oct. 1996.

[181] Y. Sato, "Voice conversation using evolutionary computation of prosodic control," in Intelligent Processing of Manufacturing of Materials '97, pp. 342-348, July 1997.

[182] Y. Sato, "Voice conversation using evolutionary computation of prosodic control," in Symposium on Artificial Life and its Applications, pp. 71-78, Dec. 1997. (in Japanese).

[183] R. Sawa and M. Hagiwara, "A system for evolving 3D machine structure using CG," in 16th Fuzzy System Symposium, (Akita, Japan), pp. 321-324, Sept. 2000. (in Japanese).

[184] M. Shibuya, H. Kita, and S. Kobayashi, "Generation of passmotion by hands using interactive genetic algorithms," in 26th Intelligent System Symposium of SICE, (Yokohama, Japan), Mar. 1999. (in Japanese).

[185] M. Shibuya, H. Kita, and S. Kobayashi, "Integration of multiobjective and interactive genetic algorithms and its application to animation design," in IEEE Int. Conf. on System, Man, and Cybernetics (SMC'99), (Tokyo, Japan), pp. 646-651, Oct. 1999. vol.3.

[186] H. Shiraki and H. Saito, "An interactive image retrieval system using genetic algorithms," in Int. Conf. on Virtual Systems and MultiMedia (VSMM'96), (Gifu, Japan), pp. 257-262, Sept. 1996.

[187] K. Sims, "Artificial evolution for computer graphics," Computer Graphics, ACM SIGGRAPH Conf. Proc., vol. 25, pp. 319-328, July/Aug. 1991.

[188] K. Sims, "Interactive evolution of dynamical systems," in 1st European Conf. on Artificial Life (F. J. Varela and P. Bourgine, eds.), (Paris, France), MIT Press, Dec. 1991. 
[189] K. Sims, "Interactive evolution of equations for procedural models," The Visual Computer, vol. 9, no. 8, pp. 466-476, 1993.

[190] J. R. Smith, "Designing biomorphs with an interactive genetic algorithm," in 4th Int. Conf. on Genetic Algorithms (ICGA'91), (San Diego, CA, USA), pp. 535-538, CA, USA: Morgan Kaufmann Publishers, July 1991.

[191] H. P. S. Snijder and R. Daru, "GECAD: A creativity supporting sketching tool for genetic layout generation," in 14th Int. Congress on Cybernetics, (Namur, Belgium), pp. 87-92, Aug. 1995.

[192] F. Sugimoto, K. Nishio, and N. Honda, "A method of fitness assignment in interactive genetic algorithm using fuzzy reasoning based on psychological measure," J. of Japanese Society for Artificial Intelligence, vol. 13, no. 5, pp. 739-745, 1998. (in Japanese).

[193] F. Sugimoto and N. Honda, "A human interface to search and draw facial images in mind by using psychometrical space model of faces," in IEEE Int. Conf. on Fuzzy Systems (FUZZIEEE'99), vol. 3, (Seoul, Korea), pp. 1585-1590, Aug. 1999.

[194] F. Sugimoto and M. Yoneyama, "Robustness against instability of sensory judgment in a human interface to draw a facial image using a psychometrical space model," in IEEE Int. Conf. on Multimedia and Expo. (ICME2000), vol. 2, (New York, NY, USA), pp. 635-638, July/Aug. 2000.

[195] Y. Sun and Z. Wang, "Interactive algorithm of large scale multiobjective 0-1 linear programming," in Large Scale Systems: Theory and Applications 1992. 6th IFAC/IFORS/IMACS Symposium, (Beijing, China), pp. 83-86, Oxford, UK: Pergamon, August 1992.

[196] M. Tabuchi and T. Taura, "Methodology for interactive knowledge acquisition between genetic learning engine and human," J. of Japan Society for Artificial Intelligence, vol. 11, no. 4, pp. 600-607, 1996. (in Japanese).

[197] H. Takagi and K. Ohya, "Improvement of input interface for interactive genetic algorithms and its evaluation," in IEICE D-156, p. 156, Mar. 1996. (in Japanese).

[198] H. Takagi and K. Ohya, "Discrete fitness values for improving the human interface in an interactive GA," in IEEE 3rd Int. Conf. on Evolutionary Computation (ICEC'96), (Nagoya, Aichi, Japan), pp. 109-112, May 1996

[199] H. Takagi, K. Ohya, and M. Ohsaki, "Improvement of input interface for interactive genetic algorithms and its evaluation," in 12th Fuzzy System Symposium, (Tokyo, Japan), pp. 513-516, June 1996. (in Japanese).

[200] H. Takagi, "System optimization without numerical target," in 1996 Biennial Conf. of the North American Fuzzy Information Processing Society (NAFIPS'96), (Berkeley, CA, USA), pp. 351-354, June 1996.

[201] H. Takagi, K. Ohya, and M. Ohsaki, "Improvement of input interface for interactive GA and its evaluation," in Int. Conf. on Soft Computing (IIZUKA'96), (Iizuka, Fukuoka, Japan), pp. 490-493, World Scientific, October and September 1996.

[202] H. Takagi, "Interactive GA for system optimization: Problems and solution," in 4th European Congress on Intelligent Techniques and Soft Computing (EUFIT'96), (Aachen, Germany), pp. 1440-1444, Sept. 1996.

[203] H. Takagi, T. Unemi, and T. Terano, "Perspective on interactive evolutionary computing," J. of Japanese Society for Artificial Intelligence, vol. 13, no. 5, pp. 692-703, 1998. (in Japanese).

[204] H. Takagi and K. Aoki, "Interactive evolutionary computation: from creativity support to engineering applications," in Workshop on Interactive Evolutionary Computation, (Fukuoka, Japan), pp. 1-6, Mar. 1998. (in Japanese).

[205] H. Takagi, M. Ohsaki, and T. Ingu, "The methods to reduce the burden of human interactive EC operators," in Workshop on Interactive Evolutionary Computation, (Fukuoka, Japan), pp. 47-52, Mar. 1998. (in Japanese).

[206] H. Takagi, "Interactive evolutionary computation: System optimization based on human subjective evaluation," in IEEE Int. Conf. on Intelligent Engineering Systems (INES'98), (Vienna, Austria), pp. 1-6, September 1998.

[207] H. Takagi, "Interactive evolutionary computation - cooperation of computational intelligence and human KANSEI -," in Int. Conf. on Soft Computing (IIZUKA'98), (Iizuka, Fukuoka, Japan), pp. 41-50, World Scientific, October and September 1998.

[208] H. Takagi and M. Ohsaki, "Automatic optimization of hearing aids based on how user hears," in Annual Meeting of Acoustic Society of Japan, 1-2-18, (Tokyo, Japan), pp. 359-360, Mar. 1999. (in Japanese).

[209] H. Takagi, S. Kamohara, and T. Takeda, "Introduction of Soft computing techniques to welfare devices," in IEEE Midnight Sun Workshop on Soft Computing Methods in Industrial Applications (SMCia/99), (Kuusamo, Finland), pp. 116-121, June 1999.

[210] H. Takagi, S.-B. Cho, and T. Noda, "Evaluation of an IGAbased image retrieval system using wavelet coefficients," in IEEE Int. Conf. on Fuzzy Systems (FUZZ-IEEE'99), (Seoul, Korea), pp. 1775-1778, Aug. 1999.

[211] H. Takagi and K. Kishi, "On-line knowledge embedding for an interactive EC-based montage system," in 3rd Int. Conf. on Knowledge-Based Intelligent Information Engineering Systems (KES'99), (Adelaide, Australia), pp. 280-283, Aug./Sept. 1999.

[212] H. Takagi and M. Ohsaki, "IEC-based hearing aids fitting," in IEEE Int. Conf. on System, Man, and Cybernetics (SMC'99), (Tokyo, Japan), pp. 657-662, Oct. 1999.

[213] H. Takagi, "Auto-optimization for systems based on kanse: from art to engineering and amusement," in Fukuoka AcademyIndustry Joint Plaza'99, (Fukuoka, Japan), pp. 64-69, Oct. 1999. (in Japanese).

[214] H. Takagi, T. Noda, and S.-B. Cho, "Psychological space to hold impression among media in common for media database retrieval system," in IEEE Int. Conf. on System, Man, and Cybernetics (SMC'99), (Tokyo, Japan), pp. 263-268, Oct. 1999.

[215] H. Takagi, "Active user intervention in an EC search," in 5th Joint Conf. on Information Sciences (JCIS2000), (Atlantic City, New Jersey, USA), pp. 995-998, Feb./Mar. 2000.

[216] H. Takagi, T. Unemi, and T. Terano, "Interactive evolutionary computation," in Genetic Algorithms 4 (H. Kitano, ed.), ch. 11, pp. 325-361, Sangyo Publisher, Aug. 2000. (in Japanese).

[217] H. Takagi and M. Ohsaki, "IEC Fitting: New framework of hearing aid fitting based on computational intelligence technology and user's preference for hearing," in Poster session PB9, Int. Hearing Aid Research Conf. (IHCON2000), (Lake Tahoe, CA, USA), pp. 49-50, Aug. 2000.

[218] H. Takagi, "Computational intelligence with human capability," in Int. Symposium on Computational Intelligence (ISCI2000) (P. Sinčák and J. Vaščák, eds.), pp. 370-384, Košice, Slovakia: Springer-Verlag, Aug./Sept. 2000.

[219] H. Takagi, "Fusion technology of neural networks and fuzzy systems: A chronicled progression from the laboratory to our daily lives," Int. J. of Applied Mathematics and Computer Science, vol. 10 , no. 4, pp. 647-673, 2000.

[220] T. Tanino, M. Tanaka, and C. Hojo, "An interactive multicriteria decision making method by using a genetic algorithm," in 36th Annual Conf. of the Institute of Systems, Control and Information Engineers (ISCIE), (Kyoto, Japan), pp. 335-336, May 1992. (in Japanese).

[221] T. Tannai and M. Hagiwara, "Colorist: A color designing support system to reflect Kansei," in 2nd Annual Conf. of JSKE2000, p. 200, Sept. 2000. (in Japanese).

[222] T. Terano and Y. Ishino, "Data analysis using simulated breeding and inductive learning methods," in IJCAI'95 Workshop on Data Engineering and Inductive Learning, (Montreal, Canada), pp. 60-96, Aug. 1995.

[223] T. Terano, Y. Ishino, and K. Yoshinaga, "Integrating machine learning and simulated breeding techniques to analyze the characteristics of consumer goods," in Evolutionary Algorithms in Management Applications (J. Biethahn and V. Nissen, eds.), pp. 211-224, Springer-Verlag, 1995.

[224] T. Terano, Y. Ishira, and K. Yoshinaga, "Integrating machine learning and simulated breeding techniques to analyze the characteristics of consumer goods," in Evolutionary Algorithms in Management Applications (J. B. and. V. Nissen, ed.), pp. 211224, Springer-Verlag, 1995.

[225] T. Terano and Y. Ishino, "Marketing data analysis using inductive learning and genetic algorithms with interactive- and automated-phases," in IEEE Int. Conf. on Evolutionary Computation (ICEC'95), (Perth, WA, Australia), pp. 771-776, Nov./Dec. 1995. vol.2.

[226] T. Terano and Y. Ishino, "Knowledge acquisition from questionnaire data using simulated breeding and inductive learning methods," Expert Systems With Applications, vol. 11, no. 4, pp. 507-518, 1996. 
[227] T. Terano and Y. Ishino, "Interactive knowledge discovery from marketing questionnaire using simulated breeding and inductive learning methods," in 2nd Int. Conf. on Knowledge Discovery and Data Mining. (KDD-96), (Portland, OR, USA), pp. 279-282, Aug. 1996.

[228] T. Terano, "Evolutionary computation with interactive phases - a tutorial -," in Joint Symposium on System and Information, (Sendai, Japan), pp. 59-64, Nov. 1997. (in Japanese).

[229] T. Terano and Y. Ishino, "Interactive genetic algorithm based feature selection and its application to marketing data analysis," in Feature Extraction, Construction and Selection: A Data Mining Perspective (H. Liu and H. Motoda, eds.), pp. 393-407, Kluwer Academic Publishers, Boston, 1998.

[230] T. Terano and M. Inada, "Knowledge discovery from clinical data using interactive evolutionary computation," in 42nd Knowledge-based Systems of JSAI, vol. SIG-KBS, (Yokohama, Japan), Jan. 1999. (in Japanese).

[231] S. Todd and W. Latham, "Artificial Life or surreal art?," in 1st European Conf. on Artificial Life, pp. 504-513, MIT Press, 1992.

[232] S. Todd and W. Latham, Evolutionary Art and Computers. Harcourt, Brace, Jovanovich: Academic Press, 1992.

[233] S. Todd and W. Latham, "The mutation and growth of art by computers," in Evolutionary Design by Computers (P. Bentley, ed.), ch. 9, pp. 221-250, CA, USA: Morgan Kaufmann Publishers, 1999.

[234] Y. Todoroki and H. Takagi, "User interface of an interactive evolutionary computation for speech processing," in SOFT Kyushu Chapter Meeting, (Saga, Japan), pp. 13-16, Dec. 1999. (in Japanese).

[235] Y. Todoroki and H. Takagi, "User interface of an interactive evolutionary computation for speech processing," in 6th Int. Conf. on Soft Computing (IIZUKA2000), (Iizuka, Fukuoka, Japan), pp. 112-118, Oct. 2000.

[236] N. Tokui and H. Iba, "Composition of rhythms with interactive evolutionary computation," in 14th Annual Conf. of JSAI, (Tokyo, Japan), pp. 81-82, July 2000. (in Japanese).

[237] N. Tokui and H. Iba, "Music composition with interactive evolutionary computation," in 3rd International Conference on Generative Art (GA2000), (Milan, Italy), pp. 215-226, Dec. 2000.

[238] T. Unemi, "Genetic algorithms and computer graphics arts," J. of Japan Society for Artificial Intelligence, vol. 9, no. 4, pp. 518-523, 1994. (in Japanese).

[239] T. Unemi, "A user interface for simulated breeding using multifield," in Workshop on Interactive Evolutionary Computation, (Fukuoka, Japan), pp. 42-46, Mar. 1998. (in Japanese).

[240] T. Unemi, "A design of multi-field user interface for simulated breeding," in Asia Fuzzy System Symposium (AFSS'98), (Masan, Korea), pp. pp.489-494, June 1998.

[241] T. Unemi, "SBART2.4: Breeding 2D CG images and movies, and creating a type of collage," in 3rd Int. Conf. on Knowledgebased Intelligent Information Engineering Systems (KES799), (Adelaide, Australia), pp. pp.288-291, Aug./Sept. 1999.

[242] T. Unemi, "SBART 2.4: an IEC tool for creating 2D images, movies, and collage," in Workshop on Genetic Algorithms in Visual Art and Music, (Las Vegas, NV, USA), pp. pp.21-23, July 2000.

[243] T. Unemi and E. Nakada, "A support system for music composition based on simulated breeding," in 28th Intelligent System Symposium, pp. 141-146, Society for Instrument and Control Engineers, Mar. 2001. (in Japanese).

[244] J. Ventrella, "Explorations in the emergence of morphology and locomotion behavior in animated characters," in Artificial Life IV.: 4th Int. Workshop on the Synthesis and Simulation of Living System, (Cambridge, MA, USA), pp. 436-441, MIT Press, July 1994.

[245] J. Ventrella, "Disney meets Darwin-the evolution of funny animated figures," in Computer Animation '95, (Geneva, Switzerland), pp. 35-43, IEEE Computer Society Press, Apr. 1995.

[246] G. Venturini, M. Slimane, F. Morin, and J. P. A. de Beauville, "On using interactive genetic algorithms for knowledge discovery in databases," in 7th Int. Conf. on Genetic Algorithm (ICGA'97), pp. 696-703, CA, USA: Morgan Kaufmann Publishers, July 1997.

[247] V. Vucic and H. H. Lund, "Self-evolving arts - organisms versus fetishes," Muhely - The Hungarian J. of Modern Art, vol. 104, pp. 69-79, 1997.
48] M. Watanabe, S. Sakamoto, M. Ohsaki, and H. Takagi, "Development of an IEC fitting system for commercial hearing aids," in Technical Report of ASJ, H-2000-6, (Iwate, Japan), pp. 1-8, Jan. 2000. (in Japanese).

[249] T. Watanabe and H. Takagi, "Enhancement of distorted speech using interactive GA," in 11th Fuzzy System Symposium, (Okinawa, Japan), pp. 183-186, July 1995. (in Japanese).

[250] T. Watanabe and H. Takagi, "Recovering system of the distorted speech using interactive genetic algorithms," in IEEE Int. Conf. on Systems, Man and Cybernetics (SMC'95), vol. 1, (Vancouver, Canada), pp. 684-689, Oct. 1995.

[251] M. Witbrock and S. Neil-Reilly, "Evolving genetic art," in Evolutionary Design by Computers (P. Bentley, ed.), ch. 10, pp. 251-259, CA, USA: Morgan Kaufmann Publishers, 1999.

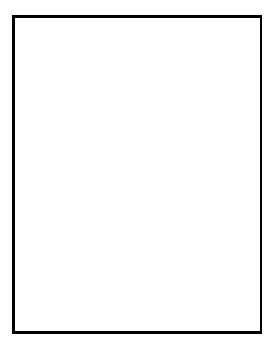

Hideyuki TAKAGI received the degrees of Bachelor and Master from Kyushu Institute of Design in 1979 and 1981, and the degree of Doctor of Engineering from Toyohashi University of Technology in 1991. His professional career includes a researcher at the Central Research Labs of Matsushita Electric Industrial Co., Ltd. in 1981 - 1995, a Visiting Industrial Fellow of the UC Berkeley in 1991 - 1993, and an Associate Professor at Kyushu Institute of Design since 1995.

Dr. Takagi is interested in the cooperative technology of neural networks, fuzzy systems, and evolutionary computation and especially in interactive evolutionary computation. He made significant contribution to the start of neuro-fuzzy system since 1988.

Dr. Takagi received the Shinohara Memorial Young Engineer Award from Institute of the Electronics, Information and Communication Engineers in 1989, the Best Paper Awards from KES'97 and IIZUKA'98 conferences in 1997 and 1998, and the PC Best Paper Awards from ICOIN-15 conference in 2001.

Dr. Takagi has served as a Chair of IEEE SMC Technical Committee on Soft Computing since 1998 and an AdCom member of the IEEE SMC Society in 2001-2003. He has also served for Japan Society for Fuzzy Theory and Systems as a Chair of Kyushu Chapter in 1997 - 1999, and a member of Executive Committee in 1999 - 2001 and 2001-2003. 